\title{
Zur Sprachdynamik des Konjunktivs im Bairischen in Österreich
}

\author{
Philipp Stöckle und Anja Wittibschlager (Wien)
}

\begin{abstract}
The paper deals with the morpho-syntactic variation of subjunctive II in Bavarian dialect regions of (former) Austria and focuses on language dynamics.

The broad empirical basis comes from two different corpora: The first corpus consists of data from the "Dictionary of Bavarian dialects in Austria" (WBÖ), reflecting the base dialects of the first half of the 20th century. These data are supplemented by recent oral data that were elicited in project part 03 "Speech repertoires and varietal spectra" of the Special Research Programme "German in Austria. Variation - Contact - Perception" (DiÖ).

Combining WBÖ and DiÖ, PP03 data, a real time comparison is carried out. For additional apparent time evidence, the internal variation within the two datasets is taken into account: While in the WBÖ data different registers are compared, variation in the DiÖ, PP03 data is analyzed with respect to different registers and different speaker groups (old vs. young).

The WBÖ and the DiÖ, PP03 data provide empirical evidence that the -ad-marker that was initially restricted to weak verbs has exceeded its domain and is universally compatible with all verb classes. In connection with this, there is a decrease of strong synthetic subjunctive II forms.

The real time as well as the apparent time findings indicate an increase of analytic subjunctive II constructions at the cost of synthetic forms. Furthermore, we find an expansion of the werdenperiphrasis, while the number of tun-subjunctives declines.
\end{abstract}

\section{$1 \quad$ Einleitung}

Im Zentrum dieses Beitrags steht der Konjunktiv II, der als besonders charakteristisch für das Bairische gilt. Neben einer bedeutenden pragmatischen Dimension, die in der Forschung häufig hervorgehoben wird (cf. z. B. Donhauser 1992; Glauninger 2011a, b), umfasst der Konjunktiv II auch in morphologisch-syntaktischer Hinsicht eine große Bandbreite an Variation, wie die folgenden Beispiele aus den Analysekorpora (WBÖ „Wörterbuch der bairischen Mundarten in Österreich“ und DiÖ, PP03 („Deutsch in Österreich, Teilprojekt 03“) (siehe Kapitel 4)) illustrieren: ${ }^{1}$

\footnotetext{
${ }^{1}$ Die Transkription der oralen DiÖ, PP03-Daten erfolgte „lautorientiert“ und ist an die Konventionen für GAT2 Minimaltranskripte angelehnt. Die WBÖ-Belegsätze wurden von den freiwilligen Sammler/-innen (siehe Kapitel 3.1) in einer an der Teuthonista orientierten Lautschrift transkribiert.
} 


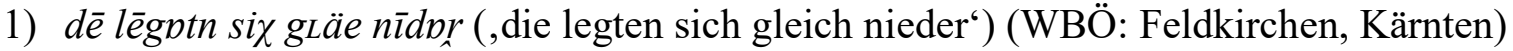

2) dann fohrad i amoi um die ganz wöt (,dann führe ich einmal um die ganze Welt') (DiÖ, PP03: Taufkirchen/Pram, Oberösterreich)

3) wenn i nu öpas saach! (,wenn ich nur etwas sähe!‘) (WBÖ: Kufstein, Nordtirol)

4) donn dad i spün (,dann täte ich spielen') (DiÖ, PP03: Taufkirchen/Pram, Oberösterreich)

5) ois bundeskonzler darad $i$ in wödfrieden herstön (,als Bundeskanzler täte ich den Weltfrieden herstellen') (DiÖ, PP03: Neumarkt/Ybbs, Niederösterreich)

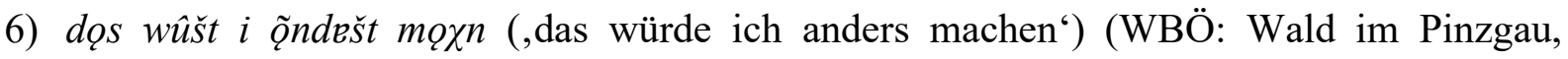
Salzburg)

In der synthetischen Form wird der Konjunktiv II typischerweise mit dem Suffix -ad gebildet, das sowohl in Kombination mit schwachen (1) als auch starken (2) Verben auftreten kann, wobei bei Letzteren ebenfalls eine Flexion mit Umlaut möglich ist (3). Daneben existiert eine analytische Konjunktivvariante, die im Dialekt vorrangig mit dem Hilfsverb tun (4) gebildet wird, zu dem in manchen Fällen zusätzlich das Suffix - ad hinzutritt (5). Während die Umschreibung mit werden (6) dialektal eher selten belegt ist, stellt sie in standardnahen Kontexten den weitaus häufigeren Fall dar.

In sprachdynamischer Hinsicht lassen sich in der Forschungsliteratur zwei Prozesse identifizieren, deren umfassende empirische Untersuchung bislang ein Desiderat darstellt: einerseits der Ersatz synthetischer durch analytische Formen, andererseits die Bildung der Umschreibung mit werden anstelle von tun.

Ziel dieses Beitrags ist, diese sprachdynamischen Prozesse für das Bairische in Österreich erstmals auf einer breiten empirischen Basis zu untersuchen und Steuerungsfaktoren herauszuarbeiten. Als basisdialektale Grundlage dienen Daten des „Wörterbuchs der bairischen Mundarten in Österreich“"(WBÖ) (cf. Stöckle 2021), die im Wesentlichen den Sprachstand in der ersten Hälfte des 20. Jahrhunderts widerspiegeln. Diesen Daten werden aktuelle Daten aus dem Teilprojekt 03 „Sprachrepertoires und Varietätenspektren“ (PP03) des Spezialforschungsbereichs „Deutsch in Österreich. Variation - Kontakt - Perzeption“ (DiÖ) aus einem Sprachproduktionsexperiment gegenübergestellt. Das DiÖ, PP03-Korpus umfasst Daten von jüngeren und älteren Sprecher/-innen und erlaubt so neben einem real time-Vergleich mit den historischen WBÖ-Daten außerdem apparent time-Analysen.

Zunächst werden die Konjunktiv-Konstruktionen im Forschungskontext diskutiert und die diesem Beitrag zugrunde liegenden Forschungsfragen formuliert (Kapitel 2). Im Anschluss daran werden die beiden Projekte und die für die Analyse verwendeten Daten vorgestellt (Kapitel 3). Den zentralen Teil dieses Beitrags bilden die Analysen (Kapitel 4), die schließlich in einer Synthese zusammengefasst werden (Kapitel 5).

\section{Der Konjunktiv II im Bairischen}

Wie die Beispiele 1-6 illustrieren, erweist sich der Konjunktiv II im Bairischen - gerade im Vergleich mit anderen Dialekten - als besonders gebräuchlich und facettenreich.

Zehetner (1985: 104) spricht von einer „morphologischen Überlegenheit“ des Bairischen gegenüber dem Standarddeutschen, wo mit Ausnahme von umlautfähigen starken Verben Kon- 
junktiv-II-Formen mit den Formen des Indikativs Präteritum zusammenfallen. Diese „Überlegenheit" des Bairischen erwächst daraus, dass das Konjunktiv-Inventar das Suffix -ad beinhaltet, das die Bildung eindeutiger Konjunktiv-II-Formen jedes Verbs erlaubt. Der -ad-Marker lässt sich auf das althochdeutsche Suffix der II. schwachen Verbklasse zurückführen (cf. Schönbach 1899: 236), ist aber mittlerweile über sein ursprüngliches Anwendungsgebiet hinaus universeller mit allen Verbklassen kompatibel (cf. Saltveit 1983: 1224). Bei der Konjunktivbildung wird das Suffix - ad bei ablautenden Verben entweder an den Präsens- (z. B. gebad) oder den Präteritumstamm (z. B. gabad) angehängt (cf. z. B. Glauninger 2011a).

Die Konstruktion mit - ad konkurriert - zumindest bei starken Verben - mit endungslosen Konjunktivformen (siehe Beispiel 2), bei denen der Konjunktiv II durch den Wechsel des Stammvokals markiert wird (z. B. geben - gab) (cf. z. B. Zehetner 1977: 117; Wiesinger 1989: 56).

Abseits dieser zwei morphologischen Bildungsmuster besteht im Bairischen auch die Option, den Konjunktiv II syntaktisch auszudrücken. Dabei fungiert vorrangig tun (siehe Beispiel 3) als Konjunktivauxiliar (cf. z. B. Wiesinger 1989: 56; Abraham/Fischer 1998; Eroms 1998; Stöckle 2020). Beide Bildungsweisen, morphologische und syntaktische, können auch in Kombination auftreten. Indem (primär) ${ }^{2}$ an das Auxiliar tun der -ad Marker angehängt wird, entsteht eine Form, die den Konjunktiv zweifach anzeigt (cf. z. B. Glauninger 2008).

Tun verkörpert allerdings nicht das einzige Konjunktivhilfsverb, auch das standardkonforme werden (siehe Beispiel 4) ist im Bairischen in Gebrauch. Über das Auftreten von Konjunktiven mit werden im Bairischen herrschen allerdings unterschiedliche Ansichten. Abraham/Fischer (1998: 37) stufen dieses Konjunktivauxiliar, das als „standarddeutsches Identifikat vermieden“ wird, als „,nicht heimisch“ in den Varietäten des Bairischen in Österreich ein. In abgeschwächter Form wird diese These auch von Lenzhofer (2017: 295) gestützt, welche die Konstruktion zwar unter Osttiroler Jugendlichen dokumentiert, allerdings in einer sehr niedrigen Frequenz. Im Kontrast dazu deuten rezente Studien (cf. z. B. Glauninger 2008; Breuer/Wittibschlager 2020) auf einen hohen Stellenwert des werden-Konjunktivs im aktuellen Sprachgebrauch auch abseits des standardnäheren Bereichs hin.

Die kontroversen Stimmen in der Literatur zur Verbreitung der werden-Periphrase lassen sich auch als Indikator für Sprachwandelerscheinungen deuten. Aktuell zeichnen sich in der Forschungsliteratur zum Konjunktiv II zwei sprachdynamische Prozesse ab: Zum einen wird generell die Ausbreitung von Konjunktivperiphrasen postuliert. Von diesem Ersatz sind in erster Linie die synthetischen Formen ohne Konjunktiv-Suffix betroffen, die lediglich noch bei einer sehr überschaubaren Anzahl an hochfrequenten starken Verben gebräuchlich sind (cf. z. B. Merkle 1975: 71; Zehetner 1985: 103). Darüber hinaus lässt sich beobachten, dass der analytische Konjunktiv II mit tun zunehmend durch die werden-Umschreibung substituiert wird. Die Ausbreitung der werden-Periphrase setzte nicht erst jüngst ein, sondern lässt sich bereits im 18 . Jahrhundert in schriftsprachlichem Material feststellen (cf. Auer 2007). Den Rückgang des tunKonjunktivs sieht Auer (2007) im Zusammenhang mit dessen Stigmatisierung (zur Stigmatisierung von tun cf. z. B. auch Eroms 1998; Langer 2001; Schwarz 2009). Bislang ist dieser

\footnotetext{
2 Die Suffigierung von werden ist zwar prinzipiell auch denkbar, spielt aber eine deutlich untergeordnetere Rolle und tritt nur in Ausnahmefällen auf (cf. Glauninger 2008).
} 
Trend allerdings vorrangig durch synchrone Beobachtungen oder apparent time-Studien gestützt. Eine umfassende empirische Untersuchung in real time, die das Ziel des vorliegenden Beitrags darstellt, steht bislang aus.

Hinsichtlich der sprachgeographischen Verortung der einzelnen Konjunktivvarianten im bairischen Sprachraum Österreichs ist das (empirisch fundierte) Wissen begrenzt und die Grammatiken zum Bairischen umfassen allenfalls sporadische Hinweise. So führt beispielsweise Wiesinger (1989) unterschiedliche regionale Varianten des -ad-Suffixes an. Rezent bieten Breuer/Wittibschlager (2020) anhand gesprochensprachlicher urbaner und ruraler Experimentdaten sowie Stöckle (2020) auf Basis jener WBÖ-Daten, die auch einen Teil des Datenpools des vorliegenden Beitrags darstellen, Einblicke zur areal-horizontalen Variation. Die Ergebnisse der beiden Studien deuten einerseits darauf hin, dass auch innerhalb des bairischen Dialektraums regionale Variation besteht, andererseits zeigt sich, dass die Parameter Urbanität und Ruralität kaum als Steuerungsfaktoren für Variation in Erscheinung treten (cf. auch Edler/Oberdorfer 2022).

Abseits des dialektalsten Pols beschäftigt sich sprachgeographische Forschung außerdem mit arealer Variation in der Standardschriftsprache (siehe z. B. Variantengrammatik, Dürscheid/Elspaß/Ziegler 2018) und in der Alltagssprache (siehe Atlas zur deutschen Alltagssprache (AdA), Elspaß/Möller 2003-).

Neben regionaler Variation rückt in der Variationslinguistik zunehmend die Erforschung des gesamten vertikalen Spektrums (von Dialekt über den „mittleren Bereich“ hin zur Standardsprache, cf. Bellmann 1983: 177) in den Fokus (cf. z. B. Breuer/Wittibschlager 2020; Breuer 2021; Lenz im Druck).

Die Beschäftigung mit dem Konjunktiv II im Bairischen reicht allerdings über die skizzierten formalen, sprachdynamischen, sprachgeographischen und vertikalen Aspekte hinaus. Die Publikationen zum Konjunktiv II der letzten Jahrzehnte widmen sich außerdem den zahlreichen Funktionen des bairischen Konjunktiv II, die über jene des Standards hinausreichen, (cf. z. B. Zehetner 1985; Donhauser 1992) und seiner pragmatischen Dimension (cf. Glauninger 2011a, b; Breuer 2021). Sowohl die Polyfunktionalität als auch die Pragmatik des Konjunktiv II stellen interessante Forschungsfelder dar. Da die Daten (wie in Kapitel 3 näher erläutert), die für die Analyse herangezogen werden, eine Auswertung nach diesen Gesichtspunkten nicht (oder nur eingeschränkt) ermöglichen, sei auf diese Dimensionen an dieser Stelle nur hingewiesen, im vorliegenden Beitrag werden sie aber nicht weiterverfolgt. Im Fokus stehen vielmehr die Konstruktionsvarianten, Sprachgeographie und Sprachdynamik. Im Detail lauten die Forschungsfragen wie folgt:

Konjunktivmarkierung: In welchen Varianten tritt der synthetische Konjunktiv auf (i. e. bei starken, schwachen, unregelmäßigen Verben; mit oder ohne -ad-Markierung)?

Konjunktivkonstruktion: Wie stellen sich das Verhältnis und die Verteilung der synthetischen und analytischen Varianten dar? Welche Hilfsverben werden verwendet?

Variationslinguistische Aspekte: Lassen sich sowohl innerhalb der Datenbestände als auch über deren Vergleich Rückschlüsse auf Sprachwandel (Ausbau des analytischen Konjunktivs, Ersatz des dialektalen tun-Konjunktivs durch standarddeutsche werden-Form) ziehen? 
Welche sprachdynamischen Prozesse lassen sich aus dem Vergleich der beiden Korpora (WBÖ, DiÖ, PP03) ableiten? Inwiefern eignen sich die Daten für einen solchen Vergleich?

\section{Projektkontexte und Datengrundlagen}

\subsection{Das Wörterbuch der bairischen Mundarten in Österreich (WBÖ)}

Die älteren, basisdialektalen Daten, die in dieser Studie verwendet werden, stammen aus dem Material des „Wörterbuchs der bairischen Mundarten in Österreich“ (WBÖ). Es handelt sich dabei um ein Langzeitprojekt, das die umfassende lexikographische Darstellung und Dokumentation der Basisdialekte in (Alt-)Österreich ${ }^{3}$ zum Ziel hat. Nach seiner Begründung im Jahr 1913 fand in den folgenden Jahrzehnten die Materialsammlung mithilfe von Fragebögen statt, die an freiwillige Sammler/-innen verschickt wurde. Ergänzt wurde das Material später um direkte Erhebungen durch professionelle Explorator/-innen, die sog. Kundfahrten, und durch Exzerpte aus unterschiedlichen gedruckten Quellen. Das Ergebnis der verschiedenen Erhebungsphasen ist auf Handzetteln im sog. „Hauptkatalog“ zusammengetragen, der mit seinen ca. 3,6 Millionen Belegen die empirische Grundlage des WBÖ darstellt.

Zur Erleichterung der lexikographischen Arbeit wurde in den 1990er-Jahren damit begonnen, die Belegzettel zu digitalisieren, i. e. deren Inhalte abzuschreiben und dabei in TUSTEP zu übertragen. Da die Bände zu den Buchstaben A, B/P und C zu diesem Zeitpunkt bereits erschienen waren, wurde mit der Arbeit ab dem Buchstaben D begonnen. Als Ergebnis dieser Digitalisierungsarbeit, die 2011 abgeschlossen wurde, liegt eine Datenbank vor, die ca. 2,4 Millionen Einträge umfasst. Nachfolgend wurde die Datenbank in XML/TEI konvertiert und ist seit 2018 über das Lexikalische Informationssystem Österreich (LIÖ) online zugänglich (cf. Stöckle/Breuer im Erscheinen).

Zwar wurden die WBÖ-Daten für die dialektlexikographische Arbeit erhoben, bei welcher der Fokus stärker auf semantischen und lautlichen Aspekten liegt als auf grammatischen. Aufgrund der großen Fülle bietet das Material jedoch genügend Möglichkeiten, sich empirisch der Variation des Konjunktiv II zu nähern. Aus sprachgeographischer Sicht kommt dem Material zugute, dass es zu jedem Beleg genaue Angaben zum Herkunftsort bzw. zur Herkunftsregion gibt. Weitere Informationen, die zu einem Großteil der Belege vorhanden sind, betreffen das Lemma, die Lautung sowie die Bedeutung. Von einigen Sammler/-innen wurden zu Verben ganze Flexionsparadigmen notiert, die häufig auch Formen im Konjunktiv enthalten (z. B. i tad flęchtn ,ich täte flechten', Leobersdorf, Niederösterreich). Von besonderem Interesse für grammatische Phänomene sind außerdem die Beispielsätze, die zu vielen Belegen enthalten sind (z. B. I derfruaßat, weñ i draußn stian müaßat, Ich erfröre, wenn ich draußen stehen müsste‘, Stainz, Steiermark). Da die WBÖ-Belegdaten zwar nicht grammatisch annotiert sind, bei der Eingabe in die Datenbank jedoch systematisch Angaben zur Flexion hinzugefügt wurden, lassen sich derartige Formen über den Suchstring ,kj““ auffinden. Für die aktuelle Analyse ergab die entsprechende Suche 3325 Fundstellen im Datenmaterial, von denen jedoch ein beträchtlicher Teil den

\footnotetext{
${ }^{3}$ Aufgrund von Straffungen wurde das Bearbeitungsgebiet im Zuge der Neukonzeption seit 2016 auf die bairischsprachigen Gebiete des heutigen Österreichs und Südtirols eingeschränkt (cf. Stöckle 2021: 17).
} 
Konjunktiv I (insbesondere in Grußformeln wie Pfiad' dī Gōd! ,Behüte dich Gott!‘, Pulkautal, Niederösterreich) betrifft.

Ein weiterer Ansatzpunkt, um Konjunktivvariation in den WBÖ-Daten zu untersuchen, ergibt sich aus den Übersetzungen, die in vielen Fällen von den Sammler/-innen zu den Beispielsätzen angefertigt wurden (z. B. des wa ma foal, wån Du a so moanąst ,Das wäre mir unangenehm, wenn du von mir das denken würdest', Fusch an der Großglocknerstraße, Salzburg). Zum einen zeigt sich an diesem Beispiel aus variationslinguistischer Sicht das interessante Phänomen, dass ein synthetischer Konjunktiv moanast durch eine analytische Konstruktion denken würdest übertragen wurde, zum anderen lassen sich über die in solchen Übersetzungen enthaltenen Lexeme würde und täte und die entsprechenden Suchstrings auch Formen im Material finden, die nicht zusätzlich durch das Kürzel „kj“ gekennzeichnet wurden. Die Suche nach dem Suchstring „würd“ ergab 862 Treffer im Datenmaterial, für „tät“" ergaben sich 680 Fundstellen. ${ }^{4}$

Für die vorliegenden Analysen werden sämtliche Belege aus dem aktuellen Bearbeitungsgebiet des WBÖ - i. e. aus Österreich und Südtirol - herangezogen, die Varianten des Konjunktiv II darstellen. ${ }^{5}$ Daraus ergibt sich eine Gesamtzahl von 1986 Belegen, deren geographische Verteilung in Abbildung 1 (am Ende von Abschnitt 3.2) dargestellt ist.

\subsection{Das Teilprojekt 03 „Sprachrepertoires und Varietätenspektren“ des Spezialfor- schungsbereichs „Deutsch in Österreich“ (DiÖ, PP03)}

Als Vergleichsbasis für die WBÖ-Daten dienen rezente, gesprochensprachliche Daten, die im Rahmen des Teilprojekts 03 (,Sprachrepertoires und Varietätenspektren“; FWF F06003; Projektleitung: Alexandra N. Lenz) des Spezialforschungsbereichs „Deutsch in Österreich“ (DiÖ) in den Jahren 2016-2018 erhoben wurden. Die Datenerhebung erfolgte an ländlichen Ortspunkten in ganz Österreich, die einerseits die Bundesländer und andererseits die dialektalen Großräume ${ }^{6}$ repräsentieren: im Ostmittelbairischen: Steyrling (Oberösterreich), Neumarkt an der Ybbs (Niederösterreich), Allentsteig (Niederösterreich) und Gaweinstal (Niederösterreich); im Westmittelbairischen: Taufkirchen an der Pram (Oberösterreich); im süd-/mittelbairischen Übergangsgebiet: Hüttschlag (Salzburg), Passail (Steiermark) und Neckenmarkt (Burgenland); im Südbairischen: Tux (Tirol), Oberwölz (Steiermark) und Weißbriach (Kärnten) und abschließend im bairisch-alemannischen Übergangsgebiet: Tarrenz (Tirol). ${ }^{7}$ Diese ruralen Erhebungsorte zeichnen sich vorrangig durch einen festen Ortskern, die Distanz zur nächsten größeren Stadt, eine Einwohnerzahl zwischen 500 und 2000 Personen und das Vorliegen eines originalen Wenkerbogens $^{8}$ aus (cf. z. B. auch Lenz et al. 2019; Lenz im Druck).

\footnotetext{
${ }^{4}$ Dabei wurden bereits alle nicht relevanten Fälle, die sich aus dieser Art der Suche ergeben (z. B. „Würdigung“ oder ,Tätigkeit“), aussortiert.

${ }^{5}$ Die in diesem Beitrag präsentierten Analysen zum WBÖ-Material stützen sich zum großen Teil auf Stöckle (2020).

${ }^{6}$ Zur Dialektraumeinteilung Österreichs cf. Wiesinger 1983.

${ }^{7}$ Das Alemannische in Vorarlberg ist in DiÖ, PP03 durch den Erhebungsort Raggal abgedeckt. Raggal bleibt allerdings, aufgrund des Bairisch-Fokus des Beitrags, unberücksichtigt.

${ }^{8}$ Die Wenkerbögen sind abzurufen über REDE.
} 
Pro Ortspunkt wurden Sprachdaten sowie soziodemographische Informationen von mindestens zehn Sprecher/-innen gesammelt, bei deren Auswahl die Parameter Autochthonie, ${ }^{9}$ Alter, Geschlecht sowie formale Bildung berücksichtigt wurden. Unter den Gewährspersonen befinden sich somit pro Erhebungsort einerseits klassische NORMs und NORFs $(60+)$ und andererseits - zahlenmäßig deutlich stärker vertreten - jüngere, mobile Sprecher/-innen (18-35), die hinsichtlich ihres formalen Bildungsgrads divergieren. ${ }^{10}$

Die Methoden zur Datenerhebung im Teilprojekt 03 des SFB DiÖ sind vielfältig und reichen von kontrollierteren Erhebungsmethoden bis hin zu freieren Gesprächssituationen. Alle Gewährspersonen nahmen an einem rund vierstündigen Erhebungsprozedere teil, im Zuge dessen nicht nur unterschiedliche Ausschnitte der individuellen sprachlichen Repertoires anvisiert, sondern auch verschiedene Datentypen (z. B. Konversationsdaten, experimentelle Daten) erlangt wurden (cf. dazu z. B. auch Lenz 2018, im Druck).

Zur Analyse des Konjunktiv II bieten sich in erster Linie Daten aus dem sogenannten Sprachproduktionsexperiment an. Das multimodale ${ }^{11}$ und computergestützte Sprachproduktionsexperiment, das gezielt zur Erhebung (morpho-)syntaktischer Phänomene gestaltet wurde, umfasst in Summe 109 Aufgaben zu 13 Primärvariablen, darunter Aufgaben zur Evokation des Konjunktiv II mit kontrafaktischer Semantik. ${ }^{12}$ Im Rahmen des Experiments wurden die Gewährspersonen angehalten, sich folgende vier hypothetische Situationen vorzustellen und sprachlich zu reagieren:

Stellen Sie sich vor, Sie können noch einmal in die Schule gehen.

Stellen Sie sich vor, Sie gewinnen morgen 10 Millionen Euro im Lotto.

Stellen Sie sich vor, Sie können ab morgen noch einmal Kind sein.

Stellen Sie sich vor, Sie können für einen Monat Bundeskanzler von Österreich sein.

Im Sprachproduktionsexperiment wurden in zwei Durchgängen (intendierter Standard und intendierter Dialekt), bei gleichbleibender Aufgabenstellung aber variierender Stimulus-Varietät, jeweils unterschiedliche vertikale Registerausschnitte der Gewährspersonen angesteuert: Ein Durchgang widmet sich dem standardnäheren Bereich und präsentiert auditive Stimuli, gesprochen von einem Nachrichtensprecher des Österreichischen Rundfunks (ORF); der zweite

\footnotetext{
${ }^{9}$ Als autochthon gelten Sprecher/-innen, die im Erhebungsort aufgewachsen sind und den Großteil ihres Lebens vor Ort verbracht haben. Außerdem muss zumindest ein Elternteil ebenso aus besagtem Ort stammen.

${ }^{10}$ Das intendierte Zielsample sah an jedem Ort jeweils zwei ältere sowie acht jüngere Gewährspersonen vor, die endgültige Anzahl an Teilnehmer/-innen pro Ortspunkt schwankt allerdings leicht, da an manchen Orten mehr als die angestrebten zehn Personen erreicht werden konnten. Um den Zielen von DiÖ, PP03 gerecht zu werden, liegt - im Kontrast zur traditionellen Dialektologie - ein Hauptaugenmerk auf der heterogeneren, mobileren, jüngeren Generation (cf. auch Lenz 2003). Den lokalen Basisdialekt fokussiert Teilprojekt 02 (cf. Vergeiner/Bülow 2022), während in Teilprojekt 03 das gesamte vertikale Spektrum, und zwar besonders von jungen Gewährspersonen, anvisiert wird.

11 Während das Konjunktiv-Experiment ausschließlich auf auditive Stimuli setzt, werden in den übrigen Aufgaben verschiedene visuelle Stimuli (Bilder/Videos) mit Audiostimuli kombiniert.

${ }^{12}$ Lenz et al. 2019 bieten eine Übersicht über ausgewählte syntaktische Variablen, die im Zentrum des DiÖ, PP03Sprachproduktionsexperiments stehen. Korecky-Kröll (2020) liefert Hinweise zum Nutzen dieser Erhebungsmethode im Bereich der Morphologie.
} 
Durchgang zielt auf den dialektnäheren Bereich ab und verwendet ortsspezifische Audiostimuli, die von einem/einer Dialektsprecher/-in des jeweiligen Erhebungsortes eingesprochen wurden.

Die Relevanz von Sprachproduktionsexperimenten zur Erhebung (morpho-)syntaktischer Phänomene, die bereits in einer Vielzahl an Studien belegt wurde (cf. z. B. Breuer/Bülow 2019; Kallenborn 2019; Lenz et al. 2019; Breuer/Wittibschlager 2020; Goryczka et al. im Erscheinen), erwächst vor allem daraus, dass linguistische Steuerungsfaktoren manipuliert werden können, eine Elizitierung quantitativ ausreichender Belege möglich ist, eine intra-individuelle Vergleichbarkeit gegeben ist und, durch den Einsatz eines computergestützten Experiments, der Einfluss der Explorator/-innen gering gehalten werden kann (zu Vor- wie auch Nachteilen der in Rede stehenden Erhebungsmethode im Detail cf. z. B. Breuer/Bülow 2019; Lenz et al. 2019; Fingerhuth/Breuer 2020).

Aufgrund der dialektalen Ausrichtung des Beitrags stehen die Daten aus dem Dialektdurchgang im Vordergrund. Für die Analysen in den Kapiteln 4.1-4.3.2 ergibt sich folgende Datengrundlage: Die vier Konjunktiv-Aufgaben evozierten 1083 Belege im Dialektdurchgang des Experiments, die sich auf 12 Erhebungsorte und 132 Gewährspersonen, wovon 35 zur älteren und 97 zur jüngeren Generation gehören, verteilen.

In Abbildung 1 ist die geographische Verteilung sowohl der WBÖ- als auch der DiÖ, PP03Daten dargestellt. ${ }^{13}$ Die Kreisgröße ist dabei relativ zur Anzahl der Belege pro Ort.

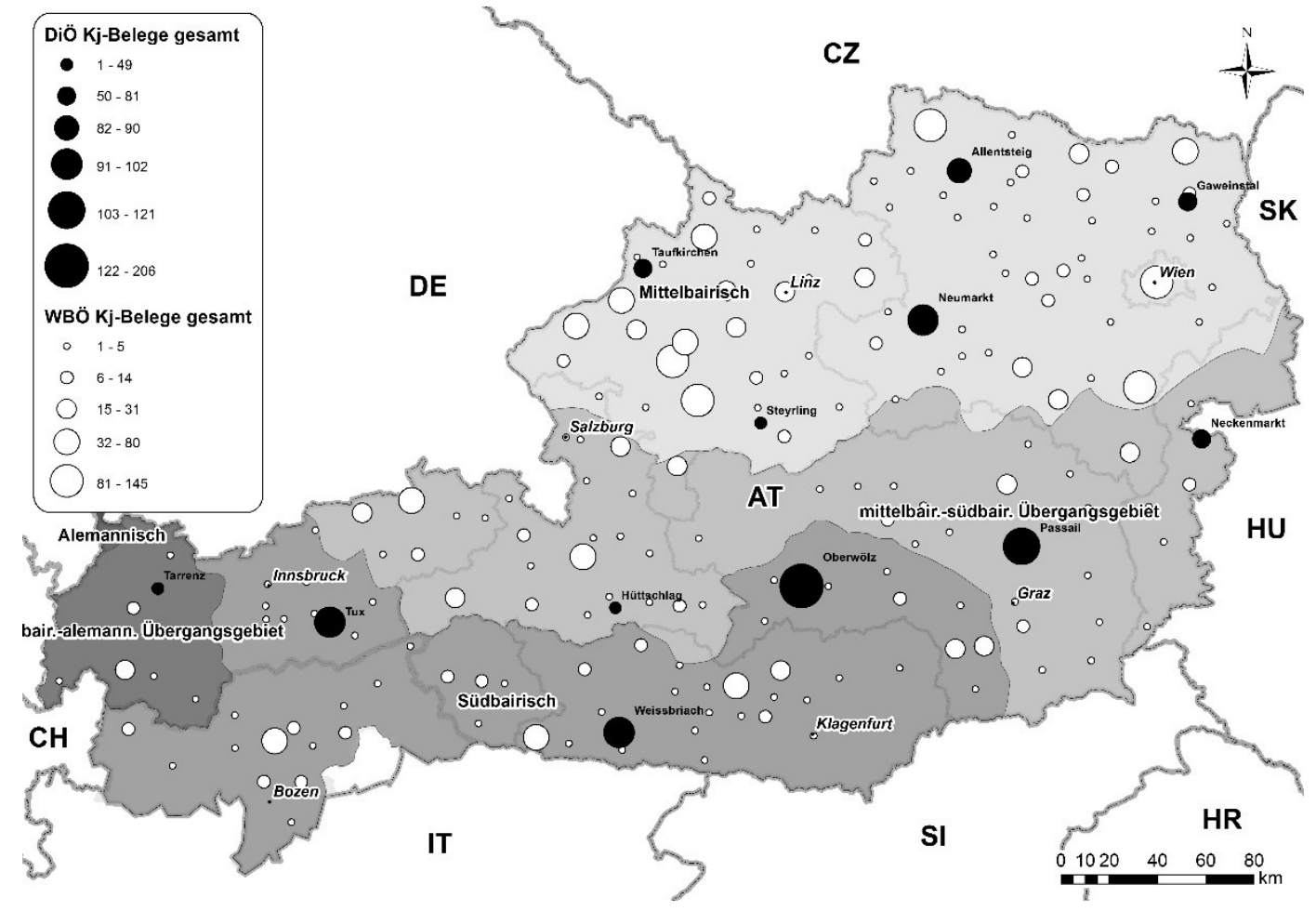

Abbildung 1: Belegzahlen pro Erhebungsort im WBÖ-Korpus (weiß) sowie im DiÖ, PP03-Korpus (schwarz) (in absoluten Zahlen)

\footnotetext{
13 Die Daten aus dem DiÖ, PP03-Standardexperiment ( $\mathrm{n}=1024$; ebenso aus 12 Orten bei 132 Sprecher/-innen) werden lediglich in Kapitel 4.3.3 präsentiert.
} 
Wie Abbildung 1 verdeutlicht, unterscheiden sich die beiden Korpora hinsichtlich der Verteilung der Daten deutlich voneinander. Dies betrifft nicht nur die unterschiedliche geographische Granularität - die Belege des DiÖ, PP03-Korpus stammen aus zwölf Orten, während sich die WBÖ-Daten auf insgesamt 187 sog. „Kleinregionen” 14 verteilen -, sondern auch die Tatsache, dass das WBÖ Daten aus Südtirol umfasst. In den folgenden vergleichenden Analysen werden diese Abweichungen daher stets Berücksichtigung finden.

\section{$4 \quad$ Analysen}

Im Folgenden werden die Analysen, die auf den in Kapitel 3.1 und 3.2 beschriebenen und in Abbildung 1 abgebildeten Daten basieren, ins Zentrum gerückt. Dafür werden zunächst die morphologische (4.1) und die syntaktische (4.2) Bildungsstrategie berücksichtigt, abschließend wird auf variationslinguistische Aspekte (4.3) eingegangen.

\subsection{Konjunktivmarkierung}

Prinzipiell lassen sich in den Daten folgende zwei Markierungstypen unterscheiden:

-ad-Markierung (waûn ă nă khâmăd, wenn er nur käme‘, Natschbach-Loipersbach, Niederösterreich)

keine -ad-Markierung (wąn ö gå nöd inta d'Laid kham, so šduəröb $\ddot{o}$ å , wenn ich gar nicht unter die Leute käme, so stürbe ich ab', Gmunden, Oberösterreich)

Weiterhin können verschiedene Flexionstypen des Verbs unterschieden werden, die jeweils mit unterschiedlichen Konjunktiv-II-Markierungen auftreten können:

schwache Verben (mîa $a^{r}$ flīchtătn uns, wir flüchteten uns', Pottendorf, Niederösterreich)

starke Verben ( i höfat eam, ich hälfe ihm', Poysdorf, Niederösterreich/wenn d' mr a bissl hulfscht, wenn du mir ein bisschen hälfest', Gnadenwald, Nordtirol)

unregelmäßige Verben ( $i$ hęd gęen e bìv , ich hätte gerne ein Bier', Herzogenburg, Niederösterreich)

Wie Abbildung $2 \mathrm{zu}$ entnehmen ist, verteilen sich Verben, die in den beiden Teilkorpora in einer (synthetischen oder analytischen) Konjunktivkonstruktion auftreten, sehr unterschiedlich auf die Verbtypen. Im WBÖ-Korpus sind starke Verben $(n=820$ bzw. 41,2 \%) am häufigsten vertreten, gefolgt von unregelmäßigen Verben $(n=693$ bzw. 34,8 \%) und schwachen Verben ( $n=473$ bzw. 23,8 \%). Im Gegensatz dazu umfassen die DiÖ, PP03-Daten in erster Linie schwache Verben $(n=543$ bzw. 50,1\%) und unregelmäßige Verben $(n=337$ bzw. 31,1\%) sowie einen geringeren Anteil an starken Verben $(n=203$ bzw. 18,7 \%). Die Differenzen zwischen den WBÖ- und den DiÖ, PP03-Verben sind statistisch signifikant ${ }^{15}$ und liegen in der Natur der Daten begründet: Während die Daten aus dem DiÖ, PP03-Projekt eher die Verteilung in der natürlichen gesprochenen Sprache widerspiegeln, lag das Ziel des WBÖs in einer möglichst vollständigen Sammlung aller (auch niederfrequenten) Verben.

\footnotetext{
${ }^{14}$ Zur geographischen Einteilung des WBÖ-Untersuchungsgebiets cf. das WBÖ-Beiheft 2 (2005) sowie das Kartentool im Lexikalischen Informationssystem Österreich (LIÖ).

${ }^{15}$ Ein $\chi^{2}$-Test nach Pearson ergibt folgendes Ergebnis: $\chi^{2}=256,51, \mathrm{df}=2, \mathrm{p}<0,001$, Cramer's $\mathrm{V}=0,29$.
} 


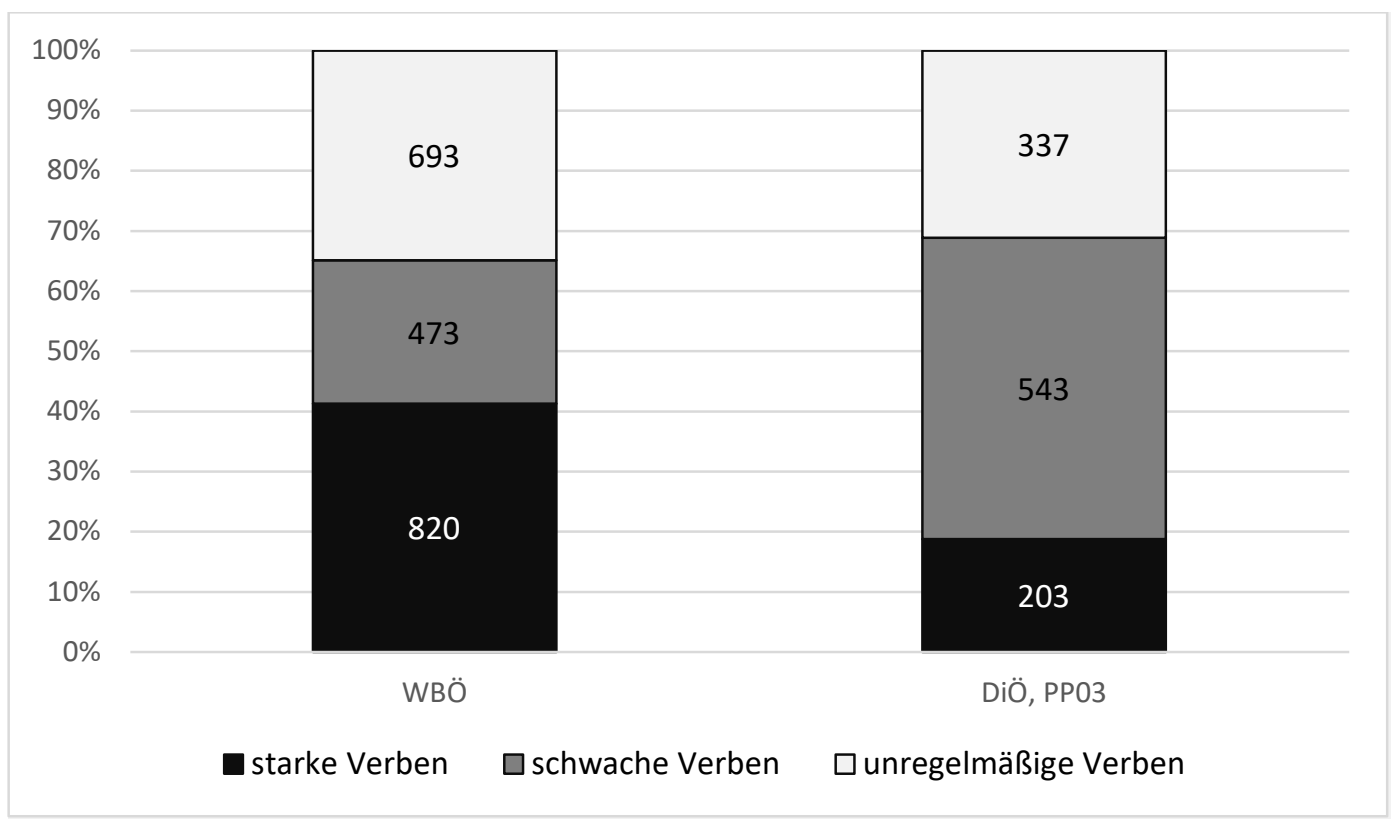

Abbildung 2: Verteilung der Verben auf Verbtypen im WBÖ- und DiÖ, PP03-Korpus

Sortiert nach dem Verbtyp visualisiert Abbildung 3 die morphologische Konjunktivmarkierung an den Vollverben wie Hilfsverben aus den beiden Korpora. Unterschieden wird dabei, ob zur Bildung des Konjunktivs der - $a d$-Marker Verwendung findet, oder ob es sich um eine Kon-

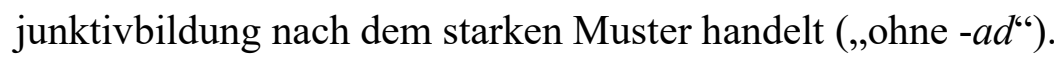

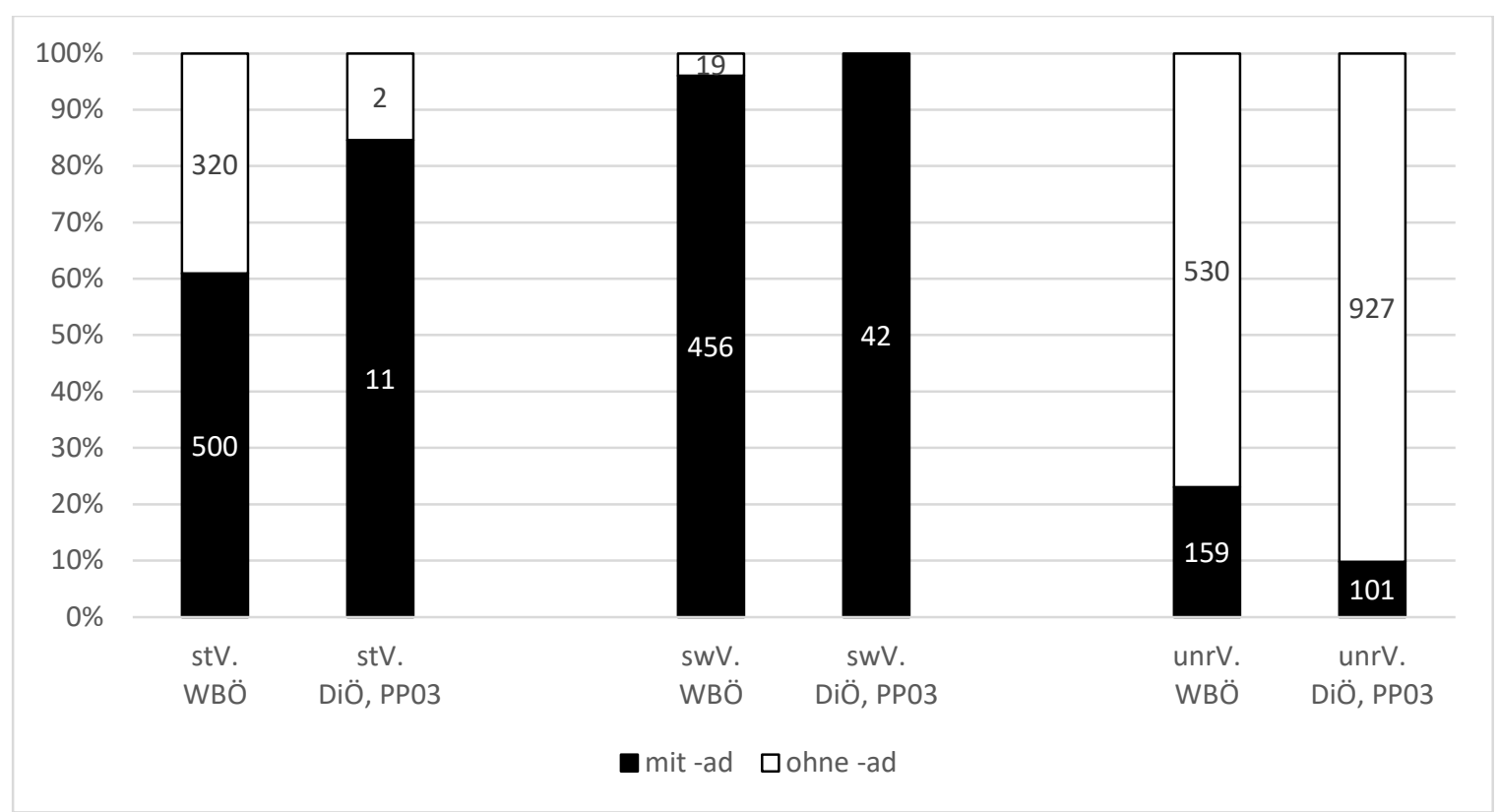

Abbildung 3: Konjunktivmarkierung an starken, schwachen und unregelmäßigen Verben im WBÖ- und DiÖ, PP03-Korpus

Über beide Korpora hinweg betrachtet, ist der -ad-Marker erwartungsgemäß bei schwachen Verben besonders verbreitet. Mit $4 \%$ aller Belege $(n=19)$ liegt im WBÖ ein verschwindend geringer Anteil an schwachen Verben im Konjunktiv ohne - $a d$ vor, im DiÖ, PP03-Korpus gibt es bei den schwachen Verben ausschließlich Konjunktivmarkierungen mit - $a d$. Bei unregelmäBigen Verben finden sich in beiden Korpora geringe Werte für die -ad-Markierung. Konjunktive ohne - $a d$ überwiegen im WBÖ-Korpus mit 76,9\% $(\mathrm{n}=530)$ und noch deutlicher im DiÖ, 
PP03-Korpus mit 90,2 \% ( $=927)$. Die Differenzen zwischen den beiden Korpora hinsichtlich der Markierung unregelmäßiger Verben kommen vorrangig durch das vermehrte Auftreten der Konjunktivauxiliare (werden und tun) in den DiÖ-Daten zustande und sind statistisch signifikant. ${ }^{16}$

Besonders hervorzuheben ist allerdings die Konjunktivmarkierung durch -ad an starken Verben. Wie in Kapitel 2 dargelegt, war der Konjunktivmarker - ad ursprünglich auf die Klasse der schwachen Verben beschränkt, erweiterte allerdings seine Domäne (cf. Pickl (2022) für eine historische Perspektive). Sowohl der Vergleich der WBÖ- und der DiÖ, PP03-Daten als auch die einzelnen Korpora für sich liefern Evidenz dafür, dass der - $a d$-Marker universeller einsetzbar ist, wobei diese Entwicklung in den DiÖ, PP03-Daten bereits weiter fortgeschritten zu sein scheint. Während sich der Anteil der Belege ohne -ad im WBÖ-Korpus noch auf 39,0 \% (n = 320) aller Belege beläuft, liegt im DiÖ, PP03-Projekt der Anteil nur noch bei 15,4 \% $(n=2) .{ }^{17}$

Im Folgenden wollen wir die Konjunktivmarkierung bei den starken Verben genauer betrachten, da bei ihnen die stärkste Variation (sowohl innerhalb der einzelnen Datensets als auch zwischen WBÖ und DiÖ, PP03) auszumachen ist, die zudem sprachhistorisch am meisten Aufschlüsse liefert. Betrachten wir die regionale Verteilung in den WBÖ-Daten, so lässt sich aus der Karte eine recht klare Tendenz herauslesen (siehe Abbildung 4).

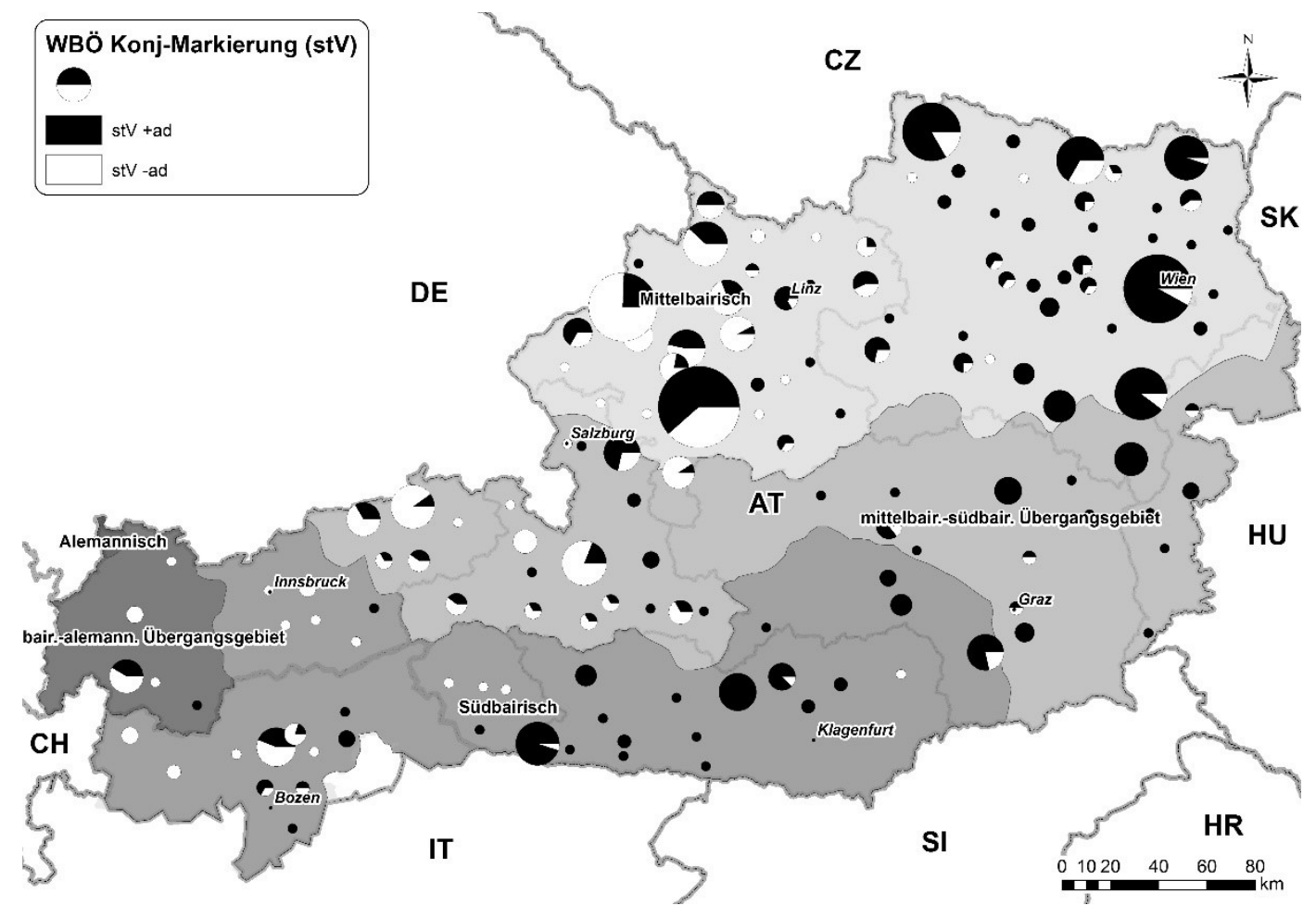

Abbildung 4: Regionale Verteilung der Konjunktivmarkierung bei den starken Verben in den WBÖ-Daten

\footnotetext{
${ }^{16}$ Ein $\chi^{2}$-Test nach Pearson ergibt folgendes Ergebnis: $\chi^{2}=55.352, \mathrm{df}=1, \mathrm{p}<0,001$, Cramer's $\mathrm{V}=0,18$.

${ }^{17}$ Aufgrund der geringen Belegzahl in den DiÖ, PP03-Daten ist dieser Unterschied jedoch nicht statistisch signifikant.
} 
Während die Variante mit -ad vor allem in Kärnten, der Steiermark, Niederösterreich und Wien belegt ist, zeigt sich eine Konzentration der Belege ohne -ad-Markierung eher in den Bundesländern, die an Deutschland grenzen. Eine Übereinstimmung mit der traditionellen Dialekteinteilung in Mittelbairisch, Südbairisch etc. lässt sich hier nicht feststellen, vielmehr vermittelt die Karte den Eindruck einer Einteilung in ein Kerngebiet, in dem die starken Flexionsformen noch erhalten sind, und einer gleichzeitigen Verbreitung der Formen mit - ad an den Rändern des deutschen Sprachraums. Die Fortsetzung dieser Tendenz bestätigt auch die Karte zur Variation des Konjunktiv II von kommen aus Renn/König (2009: 78), in der für praktisch das gesamte Mittelbairische in Bayern die Variante kaam belegt ist. Wie bereits in Stöckle (2020) bemerkt wurde, könnte diese Verteilung auf den Einfluss von Sprachkontakt zurückzuführen sein, da mit den - ad-Varianten die regelmäßig (und somit „einfacher") gebildeten Formen vermehrt in denjenigen Regionen vorkommen, in denen aufgrund der geographischen Lage mit erhöhtem Sprachkontakt zu rechnen ist.

Aufgrund der geringen Datenbasis pro Ortspunkt lassen die DiÖ, PP03-Daten keine aussagekräftigen Schlüsse hinsichtlich der regionalen Verbreitung des - $a d$-Markers in Kombination mit starken Verben innerhalb des Bairischen zu. Folgende Tendenzen sind aber abzuleiten: Beim (Mittel-)Bairischen scheint es sich um jene Region zu handeln, in der das -ad verstärkt vertreten ist, sowohl am Vollverb als auch am Auxiliar tun. Die Gesamtheit der starken Verben $(\mathrm{n}=10)$, die im bairischen Raum synthetisch realisiert wurden, weisen den -ad-Konjunktivmarker auf. In Tarrenz im bairisch-alemannischen Übergangsgebiet hingegen finden wir auch Belege ( $\mathrm{n}=$ 2) ohne - $a d$, die auf einen Einfluss des Alemannischen, wo der - $a d$-Marker - zumindest in dieser oberflächlichen Gestalt (siehe aber das Suffix - $t i$ im Alemannischen; cf. z. B. Nübling 1997) - nicht heimisch ist, hindeuten.

\subsection{Syntaktische Konstruktion}

Während sich auf morphologischer Ebene (aufgrund der teils geringen Belegzahlen in den rezenten Daten) tendenzielle Unterschiede zwischen den beiden Korpora feststellen ließen, werden Divergenzen zwischen WBÖ und DiÖ, PP03-Korpus besonders auf syntaktischer Ebene deutlich (siehe Abbildung 5). Im WBÖ zeigt sich eine starke Präferenz synthetischer Konjunktivformen (1792 Belege bzw. 90,1 \%), während Konjunktivperiphrasen mit 197 Belegen (bzw. 9,9\%) eine untergeordnete Rolle einnehmen. Konträr dazu überwiegen im DiÖ, PP03-Korpus analytische Konjunktive (815 Belege bzw. 75,3\%) deutlich und lediglich 24,7\% $(\mathrm{n}=268)$ entfallen auf synthetische Konstruktionen. Diese Unterschiede sind höchstsignifikant. ${ }^{18}$

\footnotetext{
${ }^{18}$ Ein $\chi^{2}$-Test nach Pearson ergibt folgendes Ergebnis: $\chi^{2}=1352,6, d f=1, p<0,001$, Cramer's V=0,66.
} 
P. Stöckle u. A. Wittibschlager: Zur Sprachdynamik des Konjunktivs im Bairischen in Österreich 55

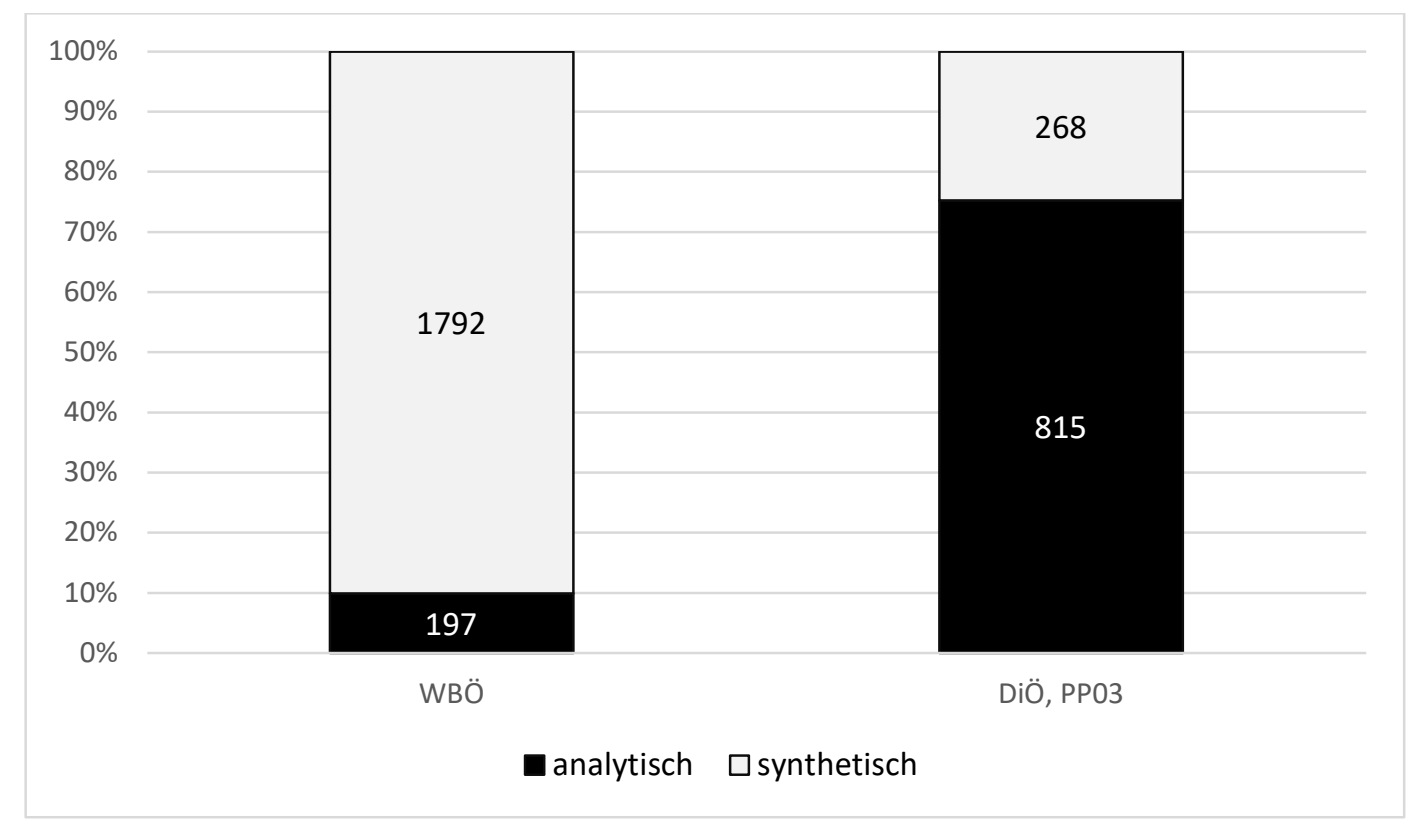

Abbildung 5: Konjunktiv-Konstruktionen im WBÖ- und DiÖ, PP03-Korpus

Wie Abbildung 6 zeigt, bestehen bei der Wahl des Konjunktivauxiliars weitere statistisch signifikante ${ }^{19}$ Differenzen zwischen den beiden Korpora. In den analytischen Konstruktionen im WBÖ fungiert vorrangig tun ( $\mathrm{n}=153$ bzw. 80,1 \%) als Konjunktivauxiliar, während die werden-Varianten mit 38 Belegen nur 19,9 \% ausmachen. Zwar tritt auch im DiÖ, PP03-Korpus tun knapp noch als häufigstes Hilfsverb auf ( $\mathrm{n}=459$ bzw. 56,3 \%), es konkurriert jedoch deutlich stärker mit werden, das in den DiÖ, PP03-Daten mit 356 Belegen bereits einen Anteil von $43,7 \%$ einnimmt.

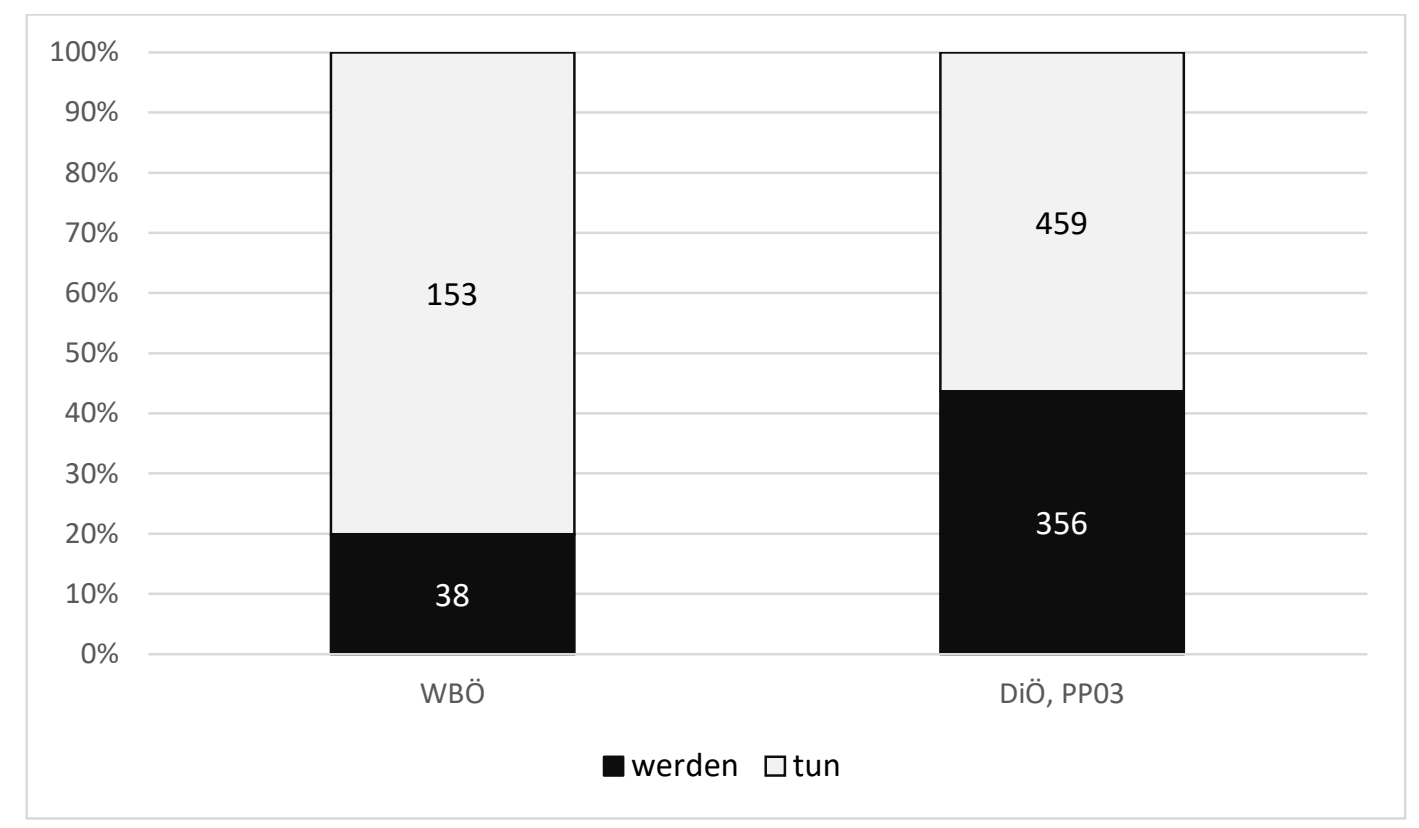

Abbildung 6: Verteilung der Konjunktivauxiliare im WBÖ- und DiÖ, PP03-Korpus

\footnotetext{
${ }^{19}$ Ein $\chi^{2}$-Test nach Pearson ergibt folgendes Ergebnis: $\chi^{2}=35,751, \mathrm{df}=1, \mathrm{p}<0,001$, Cramer's V=0,19.
} 
Im diachronen real time-Vergleich ist also eine Ausbreitung analytischer auf Kosten synthetischer Konjunktive zu verzeichnen. Unter den analytischen Konstruktionen ist es zunehmend der werden-Konjunktiv, der die Variante mit tun zu verdrängen scheint.

Hinsichtlich der arealen Verteilung synthetischer und analytischer Konjunktive in DiÖ, PP03 zeigt sich in Abbildung 7, dass die syntaktische Bildungsweise an allen Ortspunkten die präferierte Strategie darstellt. Dennoch ergibt ein verallgemeinertes lineares gemischtes Regressionsmodell (cf. Bates et al. 2015) teils signifikante Unterschiede zwischen den Orten. ${ }^{20}$ Da sich die einzelnen Ortspunkte, die sich durch höhere Anteile an synthetischen Konjunktiven auszeichnen, auf beinahe alle großen Dialektregionen Österreichs verteilen (Tux im Südbairschen, Hüttschlag und Neckenmarkt im Süd-/Mittelbairischen und Steyrling und Gaweinstal im Mittelbairischen), lässt sich daraus aber kein klares Raumbild ableiten.

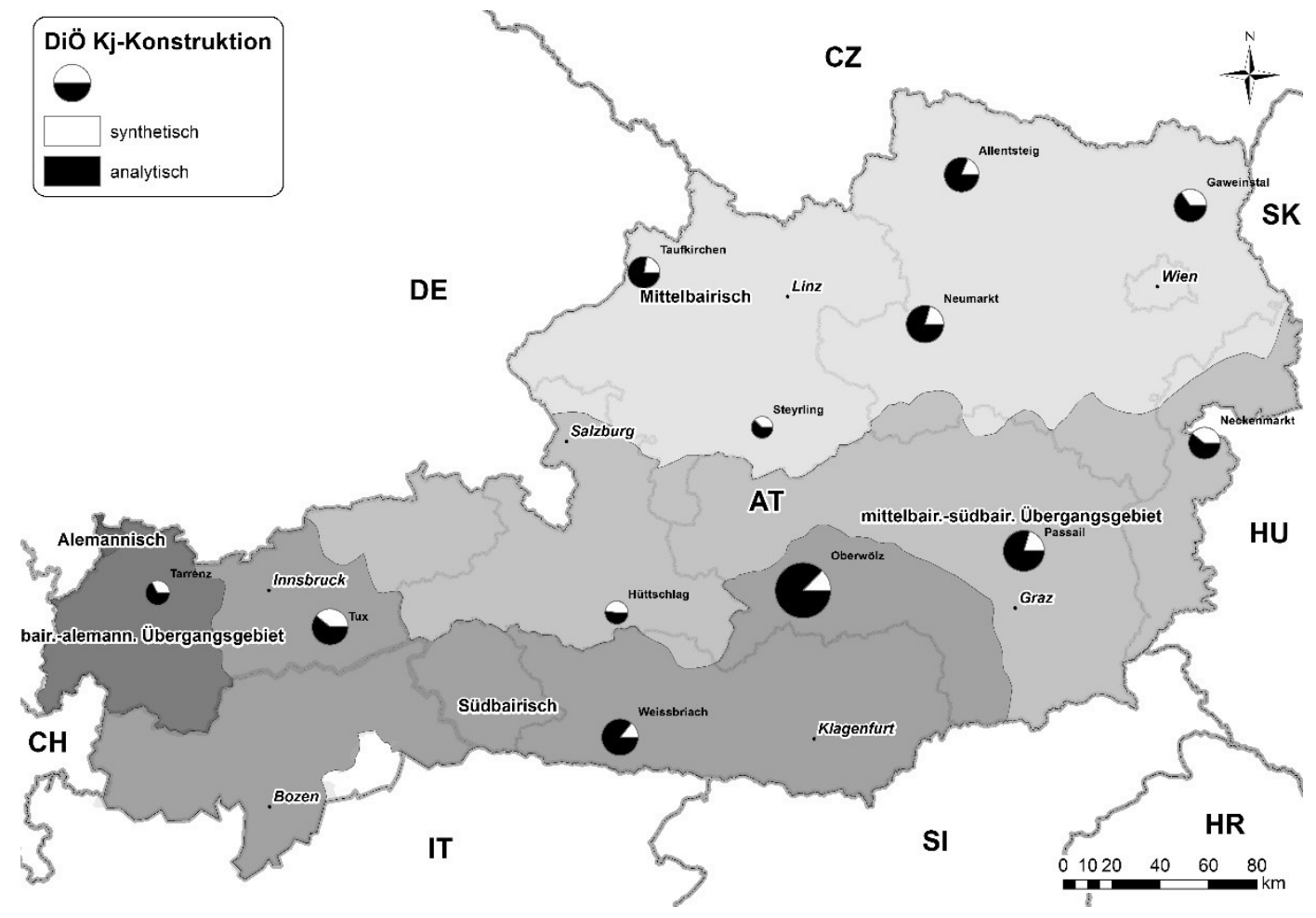

Abbildung 7: Regionale Verteilung der Konjunktivkonstruktion in den DiÖ, PP03-Daten

\subsection{Variationslinguistische Aspekte}

Abschließend steht die Variation innerhalb der jeweiligen Datensätze im Fokus, die im Kontext verschiedener sozio- und variationslinguistischer Parameter betrachtet wird und für die in diesem Zusammenhang Erklärungsansätze vorgestellt werden. Dazu zählt zum einen die Variation zwischen dialektalen Originalbelegen und den von den Sammler/-innen angefertigten standardsprachlichen Übersetzungen in den WBÖ-Daten sowie zum anderen der Vergleich verschiedener Altersgruppen und verschiedener Registerausschnitte in den DiÖ, PP03-Daten.

\footnotetext{
20 Tux: $\beta=1.017, S E=0.340, z=2.990, p=0.002$; Hüttschlag: $\beta=1.366, S E=0.405, z=3.378, p<0.001$; Neckenmarkt: $\beta=1.008, S E=0.357, z=2.828, p=0.004$; Steyrling: $\beta=0.946, S E=0.423, z=2.236, p=0.025$; Gaweinstal: $\beta=0.819$, $\mathrm{SE}=0.357, \mathrm{z}=2.298, \mathrm{p}=0.021$. AIC: 1169.5 .
} 


\subsubsection{Dialekt/Standard-Variation (WBÖ-Daten)}

Wie bereits in Abschnitt 3.1. erläutert wurde, finden sich zu einem beträchtlichen Teil der dialektalen Belegsätze Übersetzungen in den Standard bzw. standardsprachliche Paraphrasierungen. Das Verhältnis zwischen Original und Übersetzung kann dabei sehr unterschiedlich ausfallen, wie die folgenden Beispiele zeigen:

\begin{tabular}{|c|c|c|c|}
\hline Nr. & dialektales Original & Übersetzung & Muster \\
\hline 1 & $\begin{array}{l}\text { de neigiari Moasn tät am } \\
\text { gean ausfratschln }\end{array}$ & $\begin{array}{l}\text { die neugierige Meise täte ei- } \\
\text { nen gern ausfragen }\end{array}$ & täte $e_{\text {Dial }}-$ täteübers \\
\hline 2 & $i$ draat auf & ich würde aufdrehen & synth $_{\text {Dial }}-$ würdeübers \\
\hline 3 & 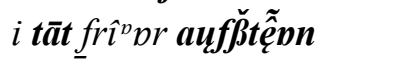 & ich würde früher aufstehen & täte $_{\text {Dial }}-$ würde Übers \\
\hline 4 & $\begin{array}{l}i \text { wušt } e p^{p} \tilde{p} n \underline{\text { frōog }} d e ̨ p \\
w \alpha \beta \text { wi } \beta^{\beta} p t\end{array}$ & $\begin{array}{l}\text { ich würde jemanden fragen, } \\
\text { der es wüßte }\end{array}$ & würde $_{\text {Dial }}-$ würde Übers \\
\hline 5 & 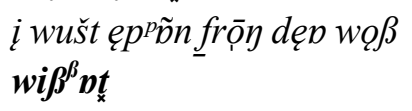 & $\begin{array}{l}\text { ich würde jemanden fragen, } \\
\text { der es wüßte }\end{array}$ & synth $_{\text {Dial }}-$ synthübers \\
\hline$\ldots$ & $\ldots$ & $\ldots$ & $\ldots$ \\
\hline
\end{tabular}

Tabelle 1: Belegsätze und deren Übersetzungen aus dem WBÖ-Material (Ausschnitt)

Es handelt sich bei den Belegsätzen nur um einen kleinen, nicht repräsentativen Ausschnitt aus dem WBÖ-Material zum Zweck der Illustration. Es lassen sich jedoch bereits hier verschiedene Muster erkennen, nach denen dialektale Belegsätze in den Standard übertragen wurden. Während in einigen Fällen Übereinstimmung vorhanden ist (Beispiele 1, 4 und 5 in Tabelle 1), finden sich in anderen Fällen Abweichungen (Beispiele 2 und 3 in Tabelle 1). Für uns sind besonders Letztere von Interesse, da hier die Frage im Hintergrund steht, wie diese Unterschiede motiviert sind und welches Normverständnis den abweichenden Übersetzungen zugrunde liegt. In Bezug auf den Konstruktionstyp lässt sich die Dialekt/Standard-Variation in den Beispielen folgendermaßen zusammenfassen:

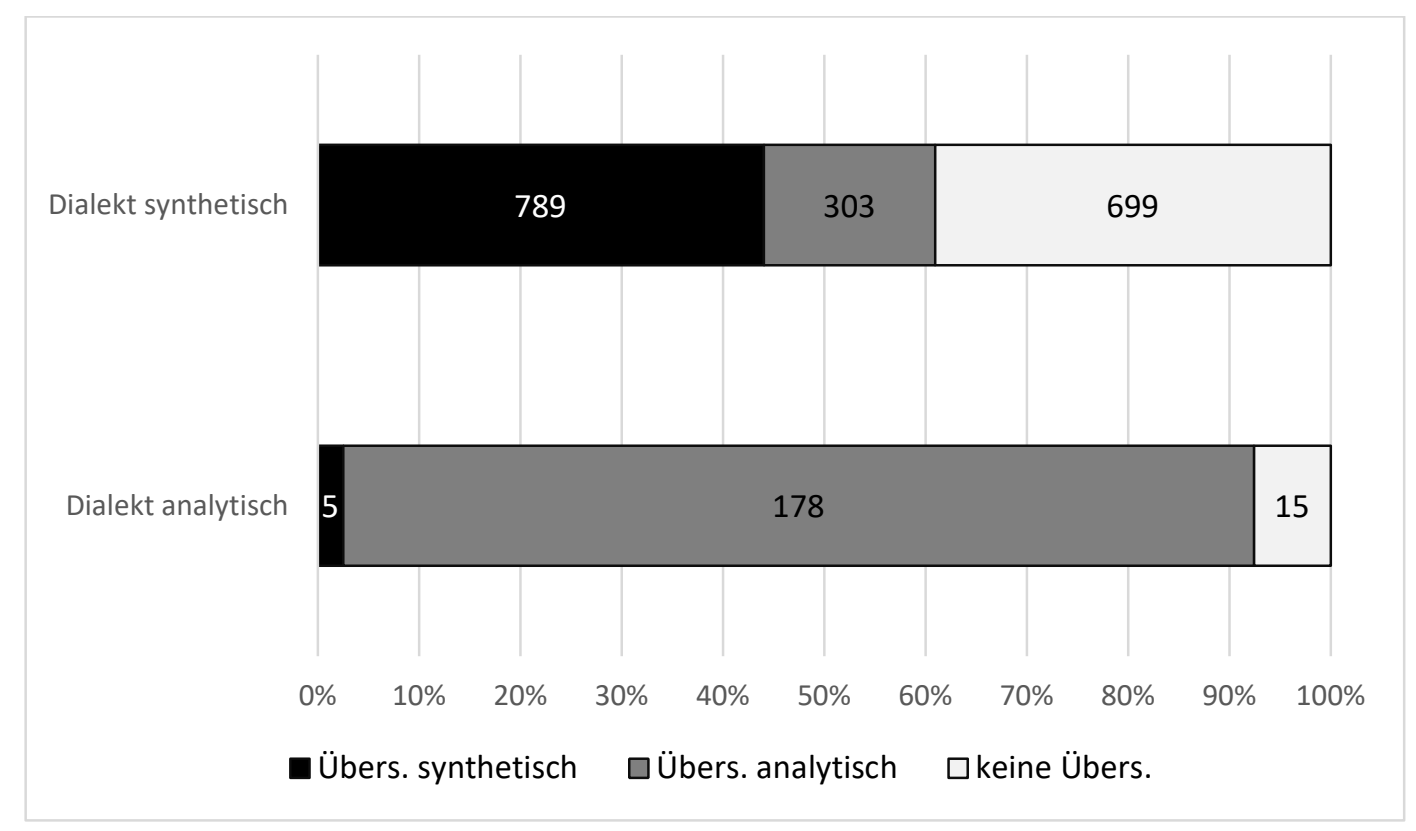

Abbildung 8: Verhältnis Konstruktionstyp Dialekt - Konstruktionstyp Übersetzung (WBÖ-Daten) 
Die beiden Balken in Abbildung 8 stehen für die Konstruktionstypen im dialektalen Original, die unterschiedlichen Graustufen geben die jeweiligen Konstruktionen in den Übersetzungen an. Von den insgesamt 1791 synthetischen Varianten des Konjunktiv II im Dialekt wurden insgesamt 789 (bzw. 44,1 \%) ebenfalls als synthetische Konstruktion übersetzt, was dem Beispiel 5 in Tabelle 1 entspricht. Von einem beträchtlichen Anteil der synthetischen Formen $(n=699$ bzw. 39,0 \%) wurde gar keine Übersetzung angefertigt. Bemerkenswert ist außerdem, dass in 303 Fällen (bzw. 16,9\%) im Standard eine analytische Konstruktion und somit der von der Vorlage abweichende Konstruktionstyp vorliegt.

Ganz anders stellt sich das Verhältnis in den Belegsätzen dar, die im Dialekt als analytische Konstruktion realisiert sind. Insgesamt liegen 198 Sätze dieses Typs vor, von denen 178 (bzw. 89,9 \%) ebenfalls als analytische Form in den Standard übertragen wurden, während in nur 5 Fällen (bzw. 2,5\%) eine abweichende, i. e. synthetische Konstruktion im Standard gewählt wurde. In 15 Fällen (bzw. 7,6 \%) liegt gar keine Übersetzung vor. Die Unterschiede bezüglich des Verhältnisses von dialektaler Vorlage und standardsprachlicher Übersetzung sind zudem statistisch höchstsignifikant. ${ }^{21}$

Betrachten wir im Folgenden das Verhältnis der Hilfsverben in den analytischen Konstruktionen.

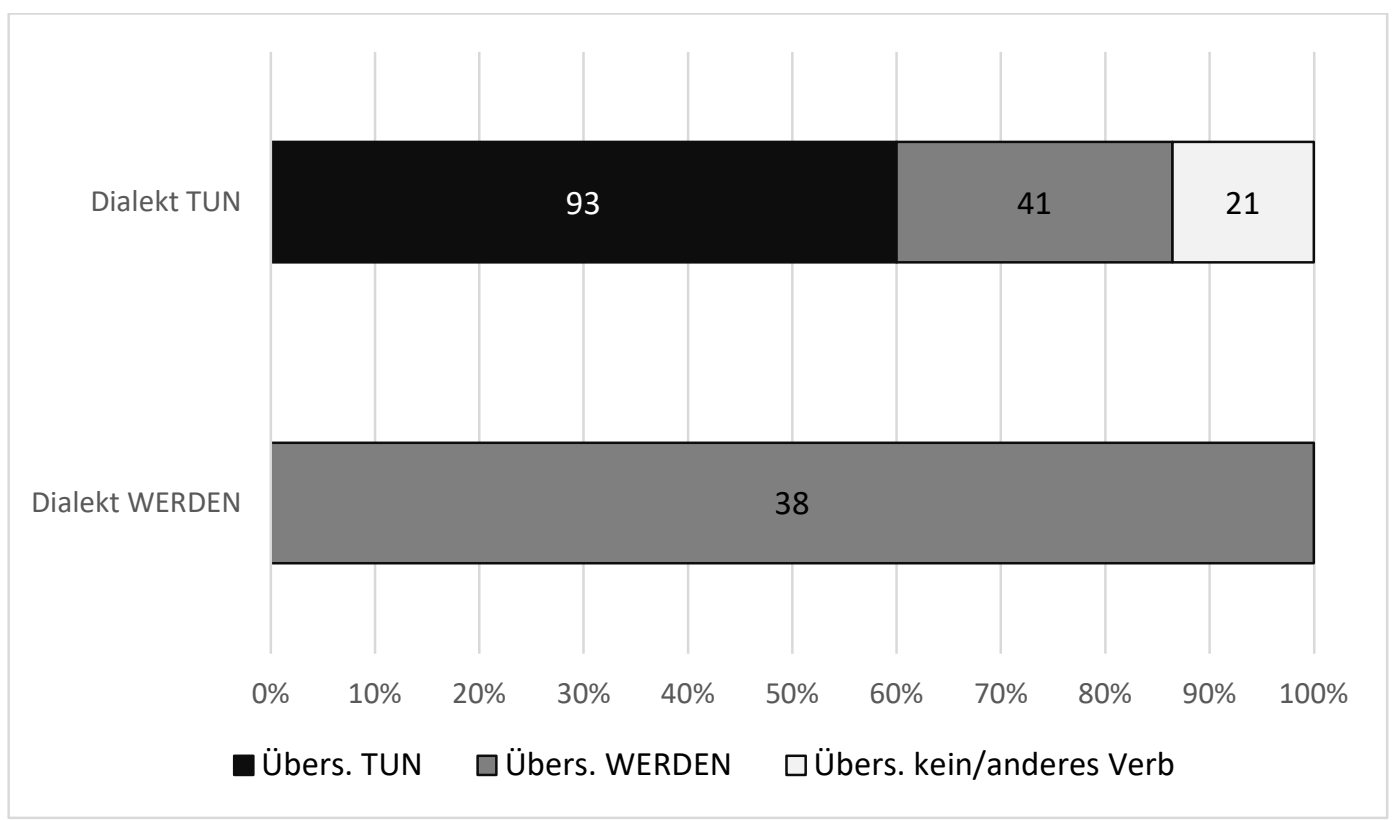

Abbildung 9: Verhältnis Hilfsverb Dialekt - Hilfsverb Übersetzung (WBÖ-Daten)

Auch hier zeigt sich ein deutlicher (und statistisch höchstsignifikanter) ${ }^{22}$ Unterschied bei den Übersetzungen in Abhängigkeit vom dialektalen Original. Von den insgesamt 155 Konstruktionen mit dem Auxiliar tun wurden 93 (bzw. 60 \%) ebenfalls mit tun in den Standard übertragen, in 41 Fällen (bzw. 26,5\%) hingegen wurde die abweichende Variante mit werden gewählt. Liegt jedoch im Dialekt bereits eine Konstruktion mit werden vor, finden sich ausschließlich Übersetzungen mit demselben Hilfsverb.

\footnotetext{
${ }^{21}$ Ein $\chi^{2}$-Test nach Pearson ergibt folgendes Ergebnis: $\chi^{2}=518,84, \mathrm{df}=2, \mathrm{p}<0,001$, Cramer's V=0,51.

22 Ein exakter Test nach Fisher ergibt einen Wert von $p<0,001$.
} 


\subsubsection{Apparent time-Vergleich (DiÖ, PP03-Korpus)}

Die Befunde aus dem real time-Vergleich hinsichtlich der Syntax gehen weitgehend mit den apparent time-Analysen innerhalb des DiÖ, PP03-Korpus konform. Die Anlage der DiÖ, PP03Erhebung ermöglicht mit zwei verschiedenen Gewährspersonengruppen (siehe Kapitel 3.2) einen Rückschluss auf Sprachwandel unter Annahme der apparent time-Hypothese ${ }^{23}$. Aus Abbildung 10 geht hervor, dass synthetische Varianten unter den älteren Gewährspersonen noch stärker vertreten sind, in der jüngeren Generation aber deutlich abnehmen. Im Hinblick auf die Periphrasen zeigt sich wiederum, dass sich insbesondere werden ausbreitet. Die Frequenz analytischer Konjunktive gebildet mit werden steigt von unter 8,4 \% $(n=19)$ bei der älteren Generation stark an auf 39,3\% $(n=337)$ bei der jüngeren Generation. Somit tritt der werdenKonjunktiv bei jüngeren Sprecher/-innen bairischer Dialekte bereits in beachtlicher Frequenz außerhalb seiner ursprünglichen Kerndomäne, dem standardnäheren Bereich, auf. Die Unterschiede zwischen den Altersgruppen bezüglich dem Konstruktionstyp sind statistisch höchstsignifikant. ${ }^{24}$

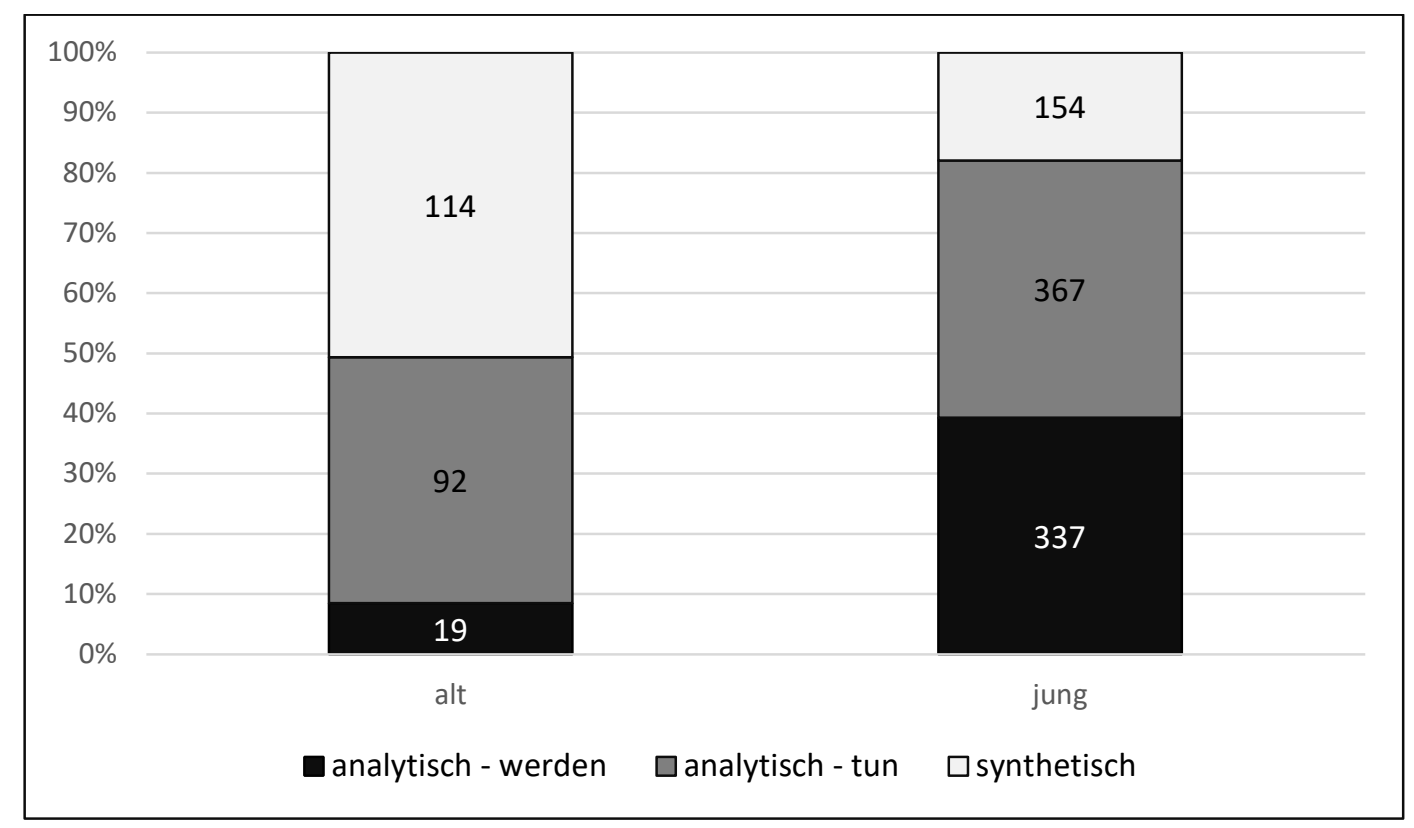

Abbildung 10: Konjunktivkonstruktion nach Altersgruppe (DiÖ, PP03-Korpus)

\subsubsection{Befunde aus dem Standard (DiÖ, PP03-Korpus)}

Da die beobachteten Sprachwandelerscheinungen auf standardsprachliche Einflüsse hinweisen, lohnt sich ein Blick in standardsprachliche Daten aus DiÖ, PP03. In beiden Durchgängen (Standarddurchgang und Dialektdurchgang) werden zwar prinzipiell die gleichen Konjunktivvarianten realisiert, dennoch zeichnen sich in Abbildung 11 markante intersituative und statistisch höchstsignifikante ${ }^{25}$ Unterschiede hinsichtlich der Frequenzen ab. Im Standarddurchgang, in dem explizit der individuell „beste“ Standard der Gewährspersonen zum Einsatz kam, treten

\footnotetext{
23 Zur apparent time-Hypothese cf. z. B. Weinreich/Labov/Herzog 1968: 184; Chambers/Trudgill 1998: $149-153$.

24 Ein $\chi^{2}$-Test nach Pearson ergibt folgendes Ergebnis: $\chi^{2}=128,81, \mathrm{df}=2, \mathrm{p}<0,001$, Cramer's V=0,35.

25 Ein exakter Test nach Fisher ergibt einen Wert von $\mathrm{p}<0,001$.
} 
lediglich jene Varianten, die der Standardnorm entsprechen, in hohen Frequenzen auf. ${ }^{26}$ Dabei werden analytische Konjunktive mit werden mit 77,8 \% $(\mathrm{n}=797)$ deutlich synthetischen Konjunktiven ohne - $a d(20,0 \%$ bzw. $\mathrm{n}=205)$ vorgezogen. Andere Varianten (-ad, tun + -ad, analytisch tun) mit jeweils Frequenzen unter 1,5\% treten nur als Randerscheinung auf und werden eher nicht dem standardnahen Bereich zugeschrieben.

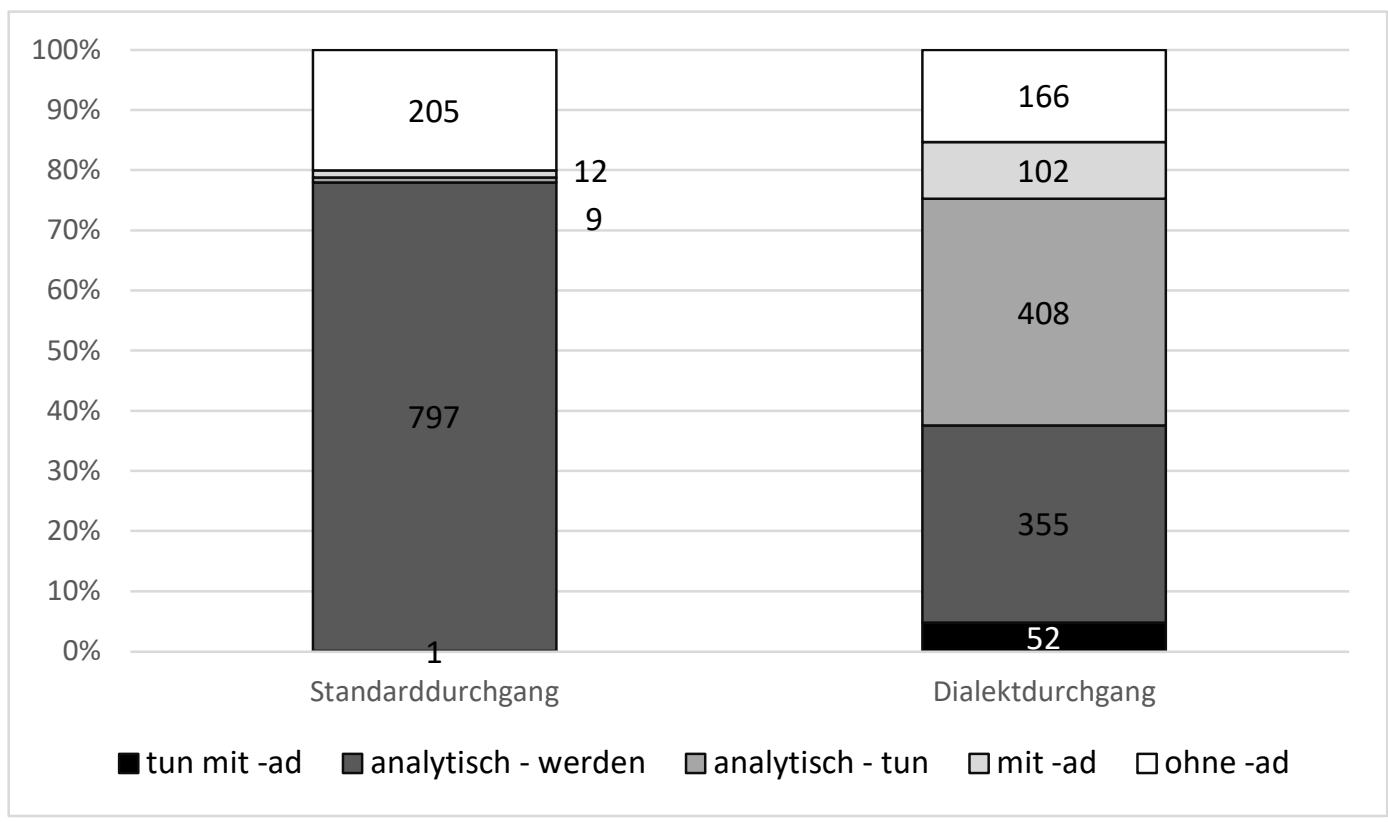

Abbildung 11: Konjunktivvarianten im Standard- und Dialektdurchgang (DiÖ, PP03-Korpus)

\section{$5 \quad$ Zusammenfassung und Synthese}

Das Ziel dieses Beitrags bestand darin, die Variation beim Konjunktiv II in den historischen WBÖ-Daten und den rezenten DiÖ, PP03-Daten in morphologischer und syntaktischer Hinsicht zu untersuchen und die Ergebnisse sprachgeographisch darzustellen und zu interpretieren. Die Variation, die sich innerhalb der einzelnen Datensätze als auch im Vergleich zeigt, diente schließlich als Grundlage dafür, Aussagen zum Sprachwandel abzuleiten.

Fasst man die verschiedenen Erscheinungsformen des Konjunktiv II (analytisch vs. synthetisch, mit - $a d$ vs. ohne - $a d$, Auxiliar werden vs. Auxiliar tun) in den jeweiligen Korpora zusammen, so zeigen sich grundlegend unterschiedliche Bilder. Dabei muss selbstverständlich berücksichtigt werden, dass sowohl bezüglich der Erhebungsmethode als auch hinsichtlich der geographischen Granularität zwischen den beiden Korpora einige Unterschiede bestehen und die Differenzen daher nur als Richtungsweiser interpretiert werden können, wohin sich der Konjunktiv II im Bairischen entwickelt. Allerdings wurde das Phänomen aus verschiedenen Perspektiven betrachtet, die in der Gesamtschau ein stimmiges und plausibles Bild der sprachdynamischen Prozesse liefern. Bevor wir uns der Synthese widmen, gehen wir noch einmal auf die Einzelergebnisse ein.

\footnotetext{
${ }^{26}$ Der konkrete Arbeitsauftrag für den Standarddurchgang des Sprachproduktionsexperiments lautete, „im besten Hochdeutsch (bzw. der im Interview genannten Bezeichnung für jene Sprachform, die ORF-Nachrichtensprecher/ -innen im Fernsehen sprechen)“ zu antworten.
} 
In Bezug auf die Konjunktivmarkierung wurde in unserer Analyse besonders die Unterscheidung nach Verbtyp hervorgehoben, da das -ad-Suffix ursprünglich aus der schwachen Verbflexion stammt, sich aber auf andere Verbtypen ausgebreitet hat. Im Vergleich der beiden Korpora konnte dabei festgestellt werden, dass die Domäne der Markierung mit - ad neben den schwachen zunehmend die starken Verben umfasst und lediglich die unregelmäßigen Verben dem „alten“, i. e. starken Bildungsmuster folgen. Während sich bei den starken Verben in den WBÖDaten immerhin noch eine räumlich-geographische Gliederung zeigt, die auf einen möglichen Einfluss von Sprachkontakt hinweist, scheint die Markierung ohne -ad bei Vollverben in den rezenten DiÖ, PP03-Daten aus dem Bairischen völlig verschwunden und findet sich lediglich im Einflussgebiet des Alemannischen.

Ein deutlicher Unterschied zwischen den Korpora zeigt sich ebenfalls in Bezug auf den Konstruktionstyp: Während die analytischen Konstruktionen im WBÖ-Korpus lediglich $10 \%$ aller Belege ausmachen, stellen sie im DiÖ, PP03-Korpus mit 75,3 \% klar die Mehrheit dar. Betrachtet man zudem das Auxiliar in den analytischen Varianten, wird eine Ausbreitung der standardnäheren Form mit werden im Vergleich zum dialektaleren tun deutlich.

Die Tendenzen, die sich im Vergleich der beiden Korpora zeigen, werden auch aufgrund der Variation deutlich, die sich innerhalb der jeweiligen Datensätze im Kontext verschiedener variationslinguistischer Parameter sprachdynamisch interpretieren lässt. In den Übersetzungen, die von den Sammler/-innen der WBÖ-Daten zu einem großen Teil der dialektalen Belegsätze angefertigt wurden, wurde sowohl eine Orientierung an synthetischen Konjunktiv-Konstruktionen als auch an werden als Auxiliar deutlich. Ebenso zeigte ein Altersgruppenvergleich in den DiÖ, PP03-Daten einen Rückgang synthetischer Konstruktionen sowie eine Ausbreitung des Auxiliars werden. Auch im Vergleich verschiedener Sprechsituationen im DiÖ, PP03-Korpus wurde die Dominanz der analytischen Konstruktion mit werden in standardnahen Registern deutlich, während sich in dialektalen Sprechlagen eine größere Vielfalt verschiedener Konjunktiv-II-Varianten zeigte.

Fasst man die Ergebnisse zusammen, lässt sich eine Entwicklung konstatieren, die insbesondere durch den kontinuierlichen Abbau unregelmäßiger Strukturen gekennzeichnet ist und verschiedene Systemebenen betrifft. Etwas vereinfacht lässt sich diese Entwicklung in vier Stufen unterteilen.

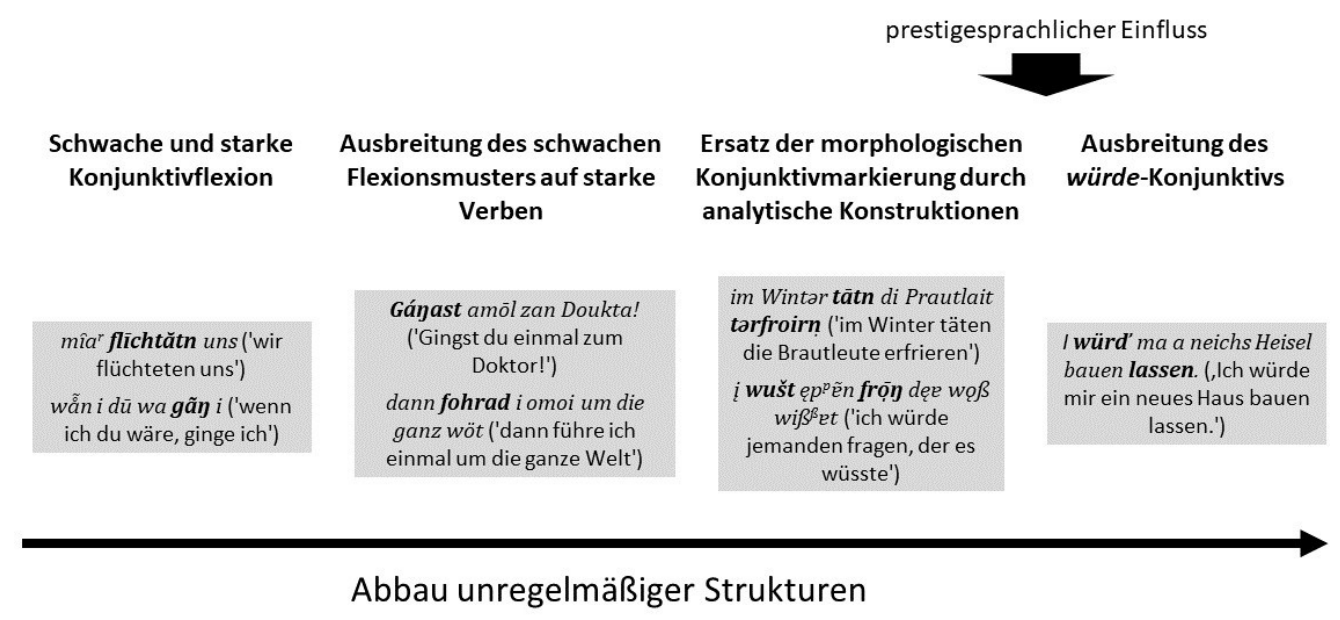

Abbildung 12: Synthese

ISSN $1615-3014$ 
Die erste Stufe, die sich anhand der Literatur belegen lässt (cf. Schönbach 1899; Wiesinger 1989) und für die es empirische Evidenz insbesondere in den WBÖ-Daten gibt, kann durch eine vergleichsweise große Vielfalt an Formen charakterisiert werden. Neben der schwachen Konjunktiv-Flexion mit dem Suffix -ad, die vor allem bei schwachen Verben auftritt, ist die starke Flexion mit Vokalwechsel bei den starken Verben verbreitet. In der zweiten Stufe wird sie jedoch zunehmend abgebaut, da sich das schwache und gleichzeitig regelmäßige Flexionsmuster auch auf die starken Verben ausbreitet.

Der Übergang von der zweiten zur dritten Stufe, die gleichfalls eine Domänenverschiebung von der Morphologie auf die Syntax darstellt, lässt sich insbesondere an der Variation zwischen WBÖ- und DiÖ, PP03-Daten nachvollziehen. Während die analytischen Formen im WBÖ-Material weitaus häufiger sind als die synthetischen, stellen letztere im DiÖ, PP03-Korpus den weitaus größeren Anteil an Konjunktiv-II-Konstruktionen dar. In der letzten Stufe schließlich findet zunehmend eine Substitution des Auxiliars tun durch werden statt. Lässt sich diese Tendenz bereits im 18. Jahrhundert als Ausdruck prestigesprachlichen Einflusses beobachten (cf. Auer 2007), stellt sie heute - besonders bei jüngeren Sprechern/-innen und in standardnäheren Sprechlagen - die häufigste Variante des Konjunktiv II dar.

Wie durch die Ausführungen in diesem Beitrag deutlich geworden ist, lässt sich dieser Prozess selbstverständlich nicht als lineare Entwicklung auffassen, sondern ist durch fließende Übergänge und Variation in den unterschiedlichen Phasen und Registern gekennzeichnet. Gleichzeitig konnte aber durch den diachronen Vergleich zweier umfangreicher Korpora - und erstmals für die Variation des Konjunktiv II im Bairischen - gezeigt werden, dass sich die für das Deutsche konstatierte allgemeine Tendenz zur Ausbreitung analytischer Formen (cf. Simon 2015: 429) und gleichsam zum Abbau unregelmäßiger Strukturen auch im Spannungsfeld bairischer Dialekte in Österreich mit der gesprochenen Standardsprache nachweisen lässt.

\section{Literaturverzeichnis}

AdA: Atlas zur deutschen Alltagssprache. Herausgegeben von Elspaß, Stephan/Möller, Robert (2003-). atlas-alltagssprache.de [12.11.2020].

Abraham, Werner/Fischer, Annette (1998): „Das grammatische Optimalisierungsszenario von tun als Hilfsverb“. In: Donhauser, Karin/Eichinger, Ludwig M. (eds.): Deutsche Grammatik - Thema in Variationen. Festschrift für Hans-Werner Eroms zum 60. Geburtstag. Heidelberg, Winter: 35-47. (= Germanistische Bibliothek 1).

Auer, Anita (2007): „Österreichisches Deutsch ist eine würde-volle Sprache“. The Subjunctive Mood in Eighteenth-Century Austria. Leiden Papers in Linguistics 4/1: 1-20.

Bates, Douglas et al. (2015): „Fitting Linear Mixed-Effects Models Using lme4.“ Journal of Statistical Software 67/1: 1-48. doi: 10.18637/jss.v067.i01.

Bellmann, Günter (1983): „Probleme des Substandards im Deutschen“. In: Mattheier, Klaus J. (ed.): Aspekte der Dialekttheorie. Tübingen, Niemeyer: 105-130.

Breuer, Ludwig M. (2021): „Wienerisch“vertikal. Syntaktische Analysen stadtsprachlicher Variation. Dissertation, Universität Wien.

Breuer, Ludwig M./Bülow, Lars (2019): „Experimental approaches in the realm of language variation - How Language Production Tests can help us to better understand language variation“. In: Bülow, Lars/Fischer, Ann-Kathrin/Herbert, Kristina (eds.): Dimensionen des 
sprachlichen Raums: Variation - Mehrsprachigkeit-Konzeptualisierung. Wien etc., Lang: 251-269. (= Schriften zur deutschen Sprache in Österreich 45). doi.org/10.3726/b15250.

Breuer, Ludwig M./Wittibschlager, Anja (2020): „On the variation of the subjunctive II in Austria - Urban and rural analyses“. Linguistic Variation 20/1: 136-171. doi.org/10.1075/ lv.19005.bre.

Chambers, Jack/Trudgill, Peter (1998): Dialectology. Cambridge: Cambridge University Press. DiÖ, PP03 = Deutsch in Österreich. Variation - Kontakt - Perzeption, Teilprojekt 03: Sprachrepertoires und Varietätenspektren. dioe.at [19.10.2020].

Donhauser, Karin (1992): „Der Konjunktiv im Bairischen“. In: Weiss, Andrea (ed.): Dialekte im Wandel. Referate der 4. Tagung zur bayerisch-österreichischen Dialektologie, Salzburg, 5. bis 7. Oktober 1989. Göppingen, Kümmerle: 226-242.

Dürscheid, Christa/Elspaß, Stephan/Ziegler, Arne (eds.) (2018): Variantengrammatik des Standarddeutschen. Ein Online-Nachschlagewerk. Verfasst von einem Autorenteam unter der Leitung von Christa Dürscheid, Stephan Elspaß und Arne Ziegler. Open-Access-Publikation. http://mediawiki.ids-mannheim.de/VarGra/index.php/Start [12.11.2020].

Edler, Stefanie/Oberdorfer, Georg (2022): „Konjunktiv-II-Variation im urbanen Sprachgebrauch in Österreich“. Linguistik online: in diesem Band.

Eroms, Hans-Werner (1998): „Periphrastic ‘tun’ present-day Bavarian and other German dialects“. In: Tieken-Boon van Ostade, Ingrid/van der Wal, Marijke/van Leuvensteijn, Arjan (eds.): DO in English, Dutch and German. History and present-day variation. Amsterdam/Münster, Stichting Neerlandistiek \& Nodus Publikationen: 139-157.

Fingerhuth, Matthias/Breuer, Ludwig M. (2020): „Language production experiments as tools for corpus construction: A contrastive study of complementizer agreement". Corpus Linguistics and Linguistic Theory. doi-org.uaccess.univie.ac.at/10.1515/cllt-2019-0075.

Glauninger, Manfred M. (2008): „Synthetische und analytische „Konjunktiv 2“-Formen im Wiener Nonstandard-Deutsch“. In: Patocka, Franz/Seiler, Guido (eds.): Dialektale Morphologie, dialektale Syntax. Beiträge zum 2. Kongress der Internationalen Gesellschaft für Dialektologie des Deutschen, Wien, 20.-23. September 2006. Wien, Praesens: 233-247.

Glauninger, Manfred M. (2011a): „Zum honorativen Konjunktiv 2 als Modalisierungsoption. Aspekte einer funktionalen Typologie des Wiener Deutsch“. In: Christen, Helen/Patocka, Franz/Ziegler, Evely (eds.): Struktur, Gebrauch und Wahrnehmung von Dialekt. Beiträge zum 3. Kongress der Internationalen Gesellschaft für Dialektologie des Deutschen (IGDD), Zürich, 7.- 9. September 2009. Wien, Praesens: 47-57.

Glauninger, Manfred M. (2011b): „Zur „(Morpho-)Pragmatik“ von „Konjunktiv 2“-Formen im Wiener Nonstandard-Deutsch“. In: Pohl, Heinz Dieter (ed.): Akten der 10. Arbeitstagung für bayerisch-österreichische Dialektologie, Klagenfurt, September 2007. Wien, Praesens: 98107. (= Klagenfurter Beiträge zur Sprachwissenschaft 34/36).

Goryczka, Pamela et al. (im Erscheinen): „Variation adnominaler Possessivkonstruktionen im Deutschen. Horizontal-areale und vertikal-soziale Analysen zum österreichischen Sprachraum“. Erscheint in: Zeitschrift für Dialektologie und Linguistik.

Kallenborn, Tim (2019): Regionalsprachliche Syntax: horizontal-vertikale Variation im Moselfränkischen. Stuttgart: Steiner Verlag. (= Zeitschrift für Dialektologie und Linguistik Beihefte 176). 
Korecky-Kröll, Katharina (2020): „Morphological dynamics of German adjective gradation in rural regions of Austria“. Zeitschrift für Dialektologie und Linguistik 87, 2020/1: 25-65.

Langer, Nils (2001): Linguistic purism in action: How auxiliary tun was stigmatized in Early New High German. Berlin/New York: de Gruyter.

Lenz, Alexandra N. (im Druck): „Sprachvariation in Österreich - Alte und neue Pfade in der Forschungslandschaft“. Erscheint in: Elspaß, Stephan/Vergeiner, Philipp/Wallner, Dominik (eds.): Struktur von Variation zwischen Individuum und Gesellschaft. (=Zeitschrift für Dialektologie und Linguistik. Beihefte).

Lenz, Alexandra N. (2003): Struktur und Dynamik des Substandards. Eine Studie zum Westmitteldeutschen (Wittlich/Eifel). Stuttgart: Steiner. (=Zeitschrift für Dialektologie und Linguistik. Beihefte 125).

Lenz, Alexandra N. (2018): ,The Special Research Programme ,German in Austria. Variation Contact - Perception““. In: Ammon, Ulrich/Costa, Marcella (eds.): Sprachwahl im Tourismus - mit Schwerpunkt Europa. Language Choice in Tourism - Focus on Europe. Choix de langues dans le tourisme - focus sur l'Europe. Berlin/Boston, de Gruyter: 269-277. (= Sociolinguistica 32).

Lenz, Alexandra N. et al. (2019): „Exploring syntactic variation by means of ,Language Production Experiments". Methods from and analyses on German in Austria“. Journal of Linguistic Geography 7/2: 63-81.

Lenzhofer, Melanie (2017): Jugendkommunikation und Dialekt: Syntax gesprochener Sprachebei Jugendlichen in Osttirol. Berlin/Boston: de Gruyter. (=Empirical Linguistics 6).

LIÖ = Lexikalisches Informationssystem Österreich. lioe.dioe.at [19.10.2020].

Merkle, Ludwig (1975): Bairische Grammatik. München: Heimeran.

Nübling, Damaris (1997): „Der alemannische Konjunktiv II zwischen Morphologie und Syntax. Zur Neuordnung des Konjunktivsystems nach dem Präteritumschwund." In: Ruoff, Arno/Peter Löffelad (eds.): Syntax und Stilistik der Alltagssprache. Beiträge der 12. Arbeitstagung zur Alemannischen Dialektologie, 25. bis 29. September 1996 in Ellwangen/Jagst. Tübingen, Niemeyer: 107-121. (=Idiomatica 18).

Pickl, Simon (2022): ,Formen und Funktionen des Konjunktivs II in historischen ostoberdeutschen Predigten. Eine Langzeit-Perspektive“. Linguistik online: in diesem Band.

REDE $=$ Regionalsprache.de. regionalsprache.de [04.11.2020].

Renn, Manfred/König, Werner (2009): Kleiner Bayerischer Sprachatlas. Mit 127 Abbildungsseiten in Farbe. 3., korrigierte und überarbeitete Aufl. München: Deutscher Taschenbuch Verlag.

Saltveit, Laurits (1983): „Anlage der Modussysteme in den deutschen Dialekten“. In: Besch, Werner et al. (eds.): Dialektologie. Ein Handbuch zur deutschen und allgemeinen Dialektforschung. Zweiter Halbband. Berlin/New York, de Gruyter: 1220-1232.

Schönbach, Anton E. (1899): „Ueber den conjunctiv praeteriti im bairisch-österreichischen“. Beiträge zur Geschichte der deutschen Sprache und Literatur 24: 232-238.

Schwarz, Christian (2009): Die ,tun'-Periphrase im Deutschen. Gebrauch und Funktion. Saarbrücken: VDM Verlag Dr. Müller.

Simon, Horst (2015): „Die Historische Syntax des Deutschen braucht die Sprachtypologie und umgekehrt“. Zeitschrift für germanistische Linguistik 43/3: 421-449. 
Stöckle, Philipp (2020): „Dialektvariation an der Schnittstelle von. Syntax, Morphologie und Lexik - Der Konjunktiv II in den Bairischen Dialekten Österreichs und Südtirols“. In: Christen, Helen et al. (eds.): Regiolekt - Der neue Dialekt? Akten des 6. Kongresses der Internationalen Gesellschaft für Dialektologie des Deutschen (IGDD). Stuttgart, Steiner: 149-173. (=Zeitschrift für Dialektologie und Linguistik. Beihefte 182).

Stöckle, Philipp (2021): „Wörterbuch der bairischen Mundarten in Österreich (WBÖ)“. In: Lenz, Alexandra N./Stöckle, Philipp (eds.): Germanistische Dialektlexikographie zu Beginn des 21. Jahrhunderts. Unter Mitarbeit von Bergermayer, Angela et al. Stuttgart, Steiner: 1146. (= Zeitschrift für Dialektologie und Linguistik. Beihefte 181).

Stöckle, Philipp/Breuer, Ludwig M. (im Erscheinen): „Das WBÖ-online im ,Lexikalischen Informationssystem Österreich“ - Zugriff und Vernetzungsmöglichkeiten“. Erscheint in: Krefeld, Thomas/Lücke, Stephan/Mutter, Christina (eds.): Berichte aus der digitalen Geolinguistik (II). Korpus im Text, Band 9. Version 6 (11.08.2020, 16:09). kit.gwi.uni-muenchen.de $/ ? \mathrm{p}=35855 \& \mathrm{v}=6$. [13.02.2020].

Vergeiner, Philip C./Bülow, Lars (2020): „Der Konjunktiv II in den ruralen Basisdialekten Österreichs. Quantitative und qualitative Befunde“. Linguistik online: in diesem Band.

WBÖ = Institut für Corpuslinguistik und Texttechnologie (ICLTT) (vormals Institut für Österreichische Dialekt- und Namenlexika) (ed.) (1970-): Wörterbuch der bairischen Mundarten in Österreich. Wien: Verlag der Österreichischen Akademie der Wissenschaften (= Bayerisch-österreichisches Wörterbuch I).

WBÖ-Beiheft 2 = Institut für österreichische Dialekt- und Namenlexika (I Dinamlex) (ed.) (2005): Wörterbuch der bairischen Mundarten in Österreich. Beiheft 2: Erläuterungen zum Wörterbuch, Lautschrift, Abkürzungsverzeichnis, Literatur- und Quellenverzeichnis, Gebietsverzeichnis (mit einer Übersichtskarte und 6 Detailkarten zum Gebietsverzeichnis). Wien: Verlag der Österreichischen Akademie der Wissenschaften.

Weinreich, Uriel/Labov, William/Herzog, Marvin I. (1968): „Empirical foundations for a Theory of Language Change“. In: Lehmann, Winfred P./Malkiel, Yakov (eds.): Directions for Historical Linguistics. Austin, University of Texas Press: 95-195.

Wiesinger, Peter (1983): „Die Einteilung der deutschen Dialekte“. In: Besch, Werner et al. (eds.): Dialektologie. Ein Handbuch zur deutschen und allgemeinen Dialektforschung. Berlin/New York, Walter de Gruyter: 807-900. (= Handbücher zur Sprach- und Kommunikationswissenschaft 1.2).

Wiesinger, Peter (1989): Die Flexionsmorphologie des Verbums im Bairischen. Wien: Verlag der ÖAW.

Zehetner, Ludwig (1977): Bairisch. Düsseldorf: Schwann. (= Dialekt, Hochsprache - kontrastiv 2).

Zehetner, Ludwig (1985): Das bairische Dialektbuch. Unter Mitarbeit von Eichinger, Ludwig M. et al. München: Beck. 